\title{
Optimal Penalty Parameters for Symmetric Discontinuous Galerkin Discretisations of the Time-Harmonic Maxwell Equations
}

\author{
D. Sármány • F. Izsák · J.J.W. van der Vegt
}

Received: 2 December 2008 / Revised: 21 December 2009 / Accepted: 12 April 2010 /

Published online: 24 April 2010

(C) The Author(s) 2010. This article is published with open access at Springerlink.com

\begin{abstract}
We provide optimal parameter estimates and a priori error bounds for symmetric discontinuous Galerkin (DG) discretisations of the second-order indefinite time-harmonic Maxwell equations. More specifically, we consider two variations of symmetric DG methods: the interior penalty DG (IP-DG) method and one that makes use of the local lifting operator in the flux formulation. As a novelty, our parameter estimates and error bounds are (i) valid in the pre-asymptotic regime; (ii) solely depend on the geometry and the polynomial order; and (iii) are free of unspecified constants. Such estimates are particularly important in three-dimensional (3D) simulations because in practice many 3D computations occur in the pre-asymptotic regime. Therefore, it is vital that our numerical experiments that accompany the theoretical results are also in 3D. They are carried out on tetrahedral meshes with high-order ( $p=1,2,3,4)$ hierarchic $H$ (curl)-conforming polynomial basis functions.
\end{abstract}

Keywords Optimal parameter estimates - Symmetric discontinuous Galerkin methods · Maxwell equations $\cdot H($ curl)-conforming vector elements

D. Sármány · F. Izsák · J.J.W. van der Vegt

Department of Applied Mathematics, University of Twente, P.O. Box 217, 7500 AE, Enschede, Netherlands

D. Sármány

e-mail: d.sarmany@math.utwente.nl

J.J.W. van der Vegt

e-mail: j.j.w.vandervegt@math.utwente.nl

F. Izsák

Eötvös Loránd University, Department of Applied Analysis and Computational Mathematics, Pázmány sétány 1/C, 1117, Budapest, Hungary

e-mail: izsakf@cs.elte.hu

D. Sármány (凶)

School of Computing, University of Leeds, LS2 9JT, Leeds, UK

e-mail: d.sarmany@leeds.ac.uk 


\section{Introduction}

The difficulties of solving the Maxwell equations usually lie in the complexity of the geometry, the presence of material discontinuities and the fact that the curl operator has a large kernel. Moreover, the unknown fields in the Maxwell equations have special geometric characteristics. These are most pronounced in the three-dimensional version of the equations, and manifest themselves in the de Rham diagram; see e.g. [6, 17, 21]. However, many of the popular numerical discretisation techniques do not satisfy the de Rham diagram at the discrete level, and often contaminate the numerical solution by producing spurious modes. One notable exception is the $H$ (curl)-conforming finite-element method, which makes use of special vector-valued polynomials to mimic the geometric properties of the electromagnetic fields at the discrete level. Based on the concept introduced by Whitney in the context of algebraic topology [31], they were proposed for the Maxwell system by Nédélec and Bossavit [5, 22, 23]. A hierarchic construction of high-order basis functions that satisfy the same properties are given in [1] for tetrahedral meshes and in [27] for more general threedimensional meshes. The fact that these functions preserve the geometric properties of the Maxwell equations has motivated many to study the Maxwell system and its numerical discretisation in the framework of differential geometry [7, 17].

However, such elements suffer from a couple of practical hurdles. In particular, although they are capable of handling complex geometrical features and material discontinuities, implementation is increasingly difficult when high-order basis functions are used. Furthermore, extending the approach to non-conforming meshes-where the local polynomial order can vary between elements and hanging nodes can be present—poses considerable difficulties.

One attractive alternative is the discontinuous Galerkin (DG) finite element method. It can handle non-conforming meshes relatively easily and the implementation of high-order basis functions is also comparatively straightforward. Research in the field of DG methods has been very active in the past ten years or so; see the recent books [13] and [16] and references therein. In the context of the Maxwell equations, a nodal approach was developed in [14], and further studied in [15]. This approach had originally been based on Lax-Friedrichs type numerical fluxes, and was later applied to the local discontinuous Galerkin method [29]. In the meantime, various DG discretisations of the low-frequency Maxwell equations [19, 20] as well as the high-frequency Maxwell equations $[9,10,18]$ have also been extensively studied. The question of spurious modes in DG discretisations has been addressed in $[9,10$, 29] for conforming meshes and, more recently, in [11] for two-dimensional non-conforming meshes.

In this work, we investigate the time-harmonic Maxwell equations in a lossless medium with inhomogeneous boundary conditions, i.e. find the (scaled) electric field $\boldsymbol{E}=\boldsymbol{E}(\boldsymbol{x})$ that satisfies

$$
\begin{aligned}
& \nabla \times \frac{1}{\mu_{\mathrm{r}}} \nabla \times \boldsymbol{E}-k^{2} \varepsilon_{\mathrm{r}} \boldsymbol{E}=\boldsymbol{J} \quad \text { in } \Omega, \\
& \boldsymbol{n} \times \boldsymbol{E}=\boldsymbol{g} \text { on } \Gamma,
\end{aligned}
$$

where $\Omega$ is an open bounded Lipschitz polyhedron in $\mathbb{R}^{3}$ with boundary $\Gamma=\partial \Omega$ and outward normal unit vector $\boldsymbol{n}$. The right-hand side $\boldsymbol{J}$ is the external source and $k$ is the (realvalued) wave number with the assumption that $k^{2}$ is not a Maxwell eigenvalue. Throughout this article the (relative) permittivity and the (relative) permeability correspond to vacuum (or dry air). That is, we set $\varepsilon_{\mathrm{r}}=1$ and $\mu_{\mathrm{r}}=1$.

Out of the many different incarnations of DG discretisations for (1) we focus on symmetric ones, simply because they provide the possibility to use linear solvers-such as MINRES - that are efficient but only applicable to symmetric matrices. The symmetric interior 
penalty DG (IP-DG) method is probably the most popular such method thanks to the simple penalisation term in the flux formulation. However, the penalisation term grows quite sharply as the polynomial order is increased or the mesh is refined. As an alternative, one may opt for a numerical flux formulation that makes use of a local lifting operator, such as the ones introduced in [4] and [8]. These formulations, together with a large number of other flux choices, were analysed in [2] for the Laplace operator, and we refer to that work and references therein for further details.

The asymptotic convergence behaviour of the IP-DG discretisation for (1) was first established in [18]. In [9], the asymptotic spectral properties of the associated eigenvalue problem

$$
\begin{aligned}
\nabla \times \frac{1}{\mu_{\mathrm{r}}} \nabla \times \boldsymbol{E}-k^{2} \varepsilon_{\mathrm{r}} \boldsymbol{E} & =\mathbf{0} \quad \text { in } \Omega, \\
\boldsymbol{n} \times \boldsymbol{E} & =\mathbf{0} \quad \text { on } \Gamma,
\end{aligned}
$$

were analysed for the IP, incomplete IP, non-symmetric IP, and local DG (LDG) methods. An a priori estimate for each of these methods results as a direct corollary of the spectral analysis.

We take a slightly different approach in this article. If the problem is three-dimensional it is often more instructive to look at the discretisation in the pre-asymptotic regime, since in many practical applications the desired error falls into that region. Such an approach was taken in [29], where it was shown that for a given mesh the discrete eigenvalues of the symmetric LDG method tend to the $H$ (curl)-conforming discrete eigenvalues as the penalty parameter tends to infinity. The same result is naturally valid for other symmetric DG discretisations, such as the ones considered here.

However, taking a too large penalty term comes at a computational cost. It results in a larger number of iterations when an iterative solver is used for the discrete linear system corresponding to (1) or (2). Furthermore, if that system is used as a semi-discrete system in time-domain computations, a large penalty term results in a particularly stringent time-step restriction for explicit time-integration methods. It is therefore essential that an optimal estimate for the penalty parameter be given that guarantees stability but does not significantly compromise computational efficiency.

An explicit expression of the IP parameter for the Poisson equations on simplicial meshes was derived in [26] and more recently in [12]. We extend these results to the Maxwell equation (1) for IP-DG and also provide an explicit expression of the DG method originally introduced in [8] as a slightly modified version of [4]. Our results are based on the trace inverse inequality [30] and on an extension of an accurate estimate for the lifting operators [25].

For our DG discretisation we use a hierarchic construction of $H$ (curl)-conforming basis functions $[1,27]$. They satisfy the global de Rham diagram in the continuous finite element setting. However, because of the discontinuous nature of the methods discussed here, we cannot expect our discretisation to be globally $H$ (curl)-conforming and to satisfy the de Rham diagram. Nevertheless, we believe that the use of $H$ (curl)-conforming basis function is beneficial, since it entails that the average across any face is also $H$ (curl)-conforming. For higher-order polynomials, it also results in a sparser stiffness matrix (i.e. discrete curl-curl operator) than standard scalar $H^{1}$-conforming basis functions.

We implement the basis functions up to order four. In principle, it is possible to increase the order further, but implementation in three dimensions is hindered by a number of practical difficulties. First, high-order (i.e. $p>9$ ) quadrature rules for tetrahedra are still 
sub-optimal and computationally expensive, making the assembly a lengthy procedure. Second, iterative solvers for indefinite linear systems are known to converge slowly, a property exacerbated by the use of very high-order $H$ (curl)-conforming basis functions.

The article is organised as follows. We define the tessellation and function spaces in Sect. 2 and derive the DG discretisation for (1) in Sect. 3. We derive explicit lower bounds for the penalty parameters in the DG methods and a priori upper bounds for the DG methods themselves in Sect. 4. Three-dimensional numerical computations are carried out in Sect. 5 to show the validity of the estimates. Finally, in Sect. 6, we conclude and provide an outlook.

\section{Tessellation and Function Spaces}

We consider a tessellation $\mathcal{T}_{h}$ that partitions the polyhedral domain $\Omega \subset \mathbb{R}^{3}$ into a set of tetrahedra $\{K\}$. Throughout the article we assume that the mesh is shape-regular and that each tetrahedron is straight-sided. The notations $\mathcal{F}_{h}, \mathcal{F}_{h}^{i}$ and $\mathcal{F}_{h}^{b}$ stand respectively for the set of all faces $\{F\}$, the set of all internal faces, and the set of all boundary faces. For a bounded domain $D \subset \mathbb{R}^{d}, d=2,3$, we denote by $H^{s}(D)$ the standard Sobolev space of functions with regularity exponent $s \geq 0$ and norm $\|\cdot\|_{s, D}$. When $D=\Omega$, we write $\|\cdot\|_{s}$. On the computational domain $\Omega$, we introduce the space

$$
H(\operatorname{curl} ; \Omega):=\left\{\boldsymbol{u} \in\left[L^{2}(\Omega)\right]^{3}: \nabla \times \boldsymbol{u} \in\left[L^{2}(\Omega)\right]^{3}\right\},
$$

with the norm $\|\boldsymbol{u}\|_{\text {curl }}^{2}=\|\boldsymbol{u}\|_{0}^{2}+\|\nabla \times \boldsymbol{u}\|_{0}^{2}$. Let $H_{0}(\operatorname{curl} ; \Omega)$ denote the subspace of $H(\operatorname{curl} ; \Omega)$ of functions with zero tangential trace. We will also use the notation $(\cdot, \cdot)_{D}$ for the standard inner product in $\left[L^{2}(D)\right]^{3}$,

$$
(\boldsymbol{u}, \boldsymbol{v})_{D}=\int_{D} \boldsymbol{u} \cdot \boldsymbol{v} \mathrm{d} V
$$

and the operator $\nabla_{h}$ for the elementwise application of $\nabla=(\partial / \partial x, \partial / \partial y, \partial / \partial z)^{T}$.

We now introduce the finite element space associated with the tessellation $\mathcal{T}_{h}$. Let $\mathcal{P}_{p}(K)$ be the space of polynomials of degree at most $p \geq 1$ on $K \in \mathcal{T}_{h}$. Over each element $K$ the $H$ (curl)-conforming polynomial space is defined as

$$
Q^{p}=\left\{\boldsymbol{u} \in\left[\mathcal{P}_{p}(K)\right]^{3} ;\left.\boldsymbol{u}_{T}\right|_{s_{i}} \in\left[\mathcal{P}_{p}\left(s_{i}\right)\right]^{2} ;\left.\boldsymbol{u} \cdot \boldsymbol{\tau}_{j}\right|_{e_{j}} \in \mathcal{P}_{p}\left(e_{j}\right)\right\},
$$

where $s_{i}, i=1,2,3,4$ are the faces of the element; $e_{j}, j=1,2,3,4,5,6$ are the edges of the element; $\boldsymbol{u}_{T}$ is the tangential component of $\boldsymbol{u}$; and $\boldsymbol{\tau}_{j}$ is the directed tangential vector on edge $e_{j}$. We define the space $\Sigma_{h}^{p}$ as

$$
\Sigma_{h}^{p}:=\left\{\sigma \in\left[L^{2}(\Omega)\right]^{3}|\sigma|_{K} \in Q^{p}, \forall K \in \mathcal{T}_{h}\right\} .
$$

Consider an interface $F \in \mathcal{F}_{h}$ between element $K^{L}$ and element $K^{R}$, and let $\boldsymbol{n}^{L}$ and $\boldsymbol{n}^{R}$ represent their respective outward pointing normal vectors. We define the tangential jump and the average of the quantity $\boldsymbol{u}$ across interface $F$ as

$$
\llbracket \boldsymbol{u} \rrbracket_{T}=\boldsymbol{n}^{L} \times \boldsymbol{u}^{L}+\boldsymbol{n}^{R} \times \boldsymbol{u}^{R} \quad \text { and } \quad\{\boldsymbol{u}\}=\left(\boldsymbol{u}^{L}+\boldsymbol{u}^{R}\right) / 2,
$$

respectively. Here $\boldsymbol{u}^{L}$ and $\boldsymbol{u}^{R}$ are the values of the trace of $\boldsymbol{u}$ at $\partial K^{L}$ and $\partial K^{R}$, respectively. At the boundary $\Gamma$, we set $\{\boldsymbol{u}\}=\boldsymbol{u}$ and $\llbracket \boldsymbol{u} \rrbracket_{T}=\boldsymbol{n} \times \boldsymbol{u}$. In case we only need the average of the tangential components, we use the notation $\{\boldsymbol{u}\}_{T}$. 
For the analysis in Sect. 4, we also define the DG norm

$$
\|\boldsymbol{v}\|_{\mathrm{DG}}=\left(\|\boldsymbol{v}\|_{0}^{2}+\left\|\nabla_{h} \times \boldsymbol{v}\right\|_{0}^{2}+\left\|\mathrm{h}^{-\frac{1}{2}} \llbracket \boldsymbol{v} \rrbracket_{T}\right\|_{0, \mathcal{F}_{h}}^{2}\right)^{\frac{1}{2}},
$$

where $\|\cdot\|_{0, \mathcal{F}_{h}}$ denotes the $L^{2}(\mathcal{F})$ norm, and $\mathrm{h}(\boldsymbol{x})=h_{F}$, which is the diameter of face $F$ containing $\boldsymbol{x}$, i.e. $\left\|\mathrm{h}^{-\frac{1}{2}} \llbracket v \rrbracket_{T}\right\|_{0, \mathcal{F}_{h}}^{2}=\sum_{F \in \mathcal{F}_{h}}\left\|h_{F} \llbracket v \rrbracket_{T}\right\|_{0, F}^{2}$. Similarly, $h_{K}$ denotes the diameter of element $K$. Note that the shape-regular property of the mesh implies that there is a positive constant $C_{d}$ independent of the mesh size such that for all faces $F$ and the associated elements $K^{R}$ and $K^{L}$ we have

$$
h_{F} \leq C_{d} \min \left\{h_{K}^{L}, h_{K^{R}}\right\} .
$$

To derive the DG formulations (in the next section) we first need to introduce global lifting operators for $\boldsymbol{u} \in \Sigma_{h}^{p}$. The global lifting operator $\mathcal{L}:\left[L^{2}\left(\mathcal{F}_{h}^{i}\right)\right]^{3} \rightarrow \Sigma_{h}^{p}$ is defined as

$$
(\mathcal{L}(\boldsymbol{u}), \boldsymbol{v})_{\Omega}=\int_{\mathcal{F}_{h}^{i}} \boldsymbol{u} \cdot \llbracket \boldsymbol{v} \rrbracket_{T} \mathrm{~d} A, \quad \forall \boldsymbol{v} \in \Sigma_{h}^{p},
$$

and the global lifting operator $\mathcal{R}:\left[L^{2}\left(\mathcal{F}_{h}\right)\right]^{3} \rightarrow \Sigma_{h}^{p}$ as

$$
(\mathcal{R}(\boldsymbol{u}), \boldsymbol{v})_{\Omega}=\int_{\mathcal{F}_{h}} \boldsymbol{u} \cdot\{\boldsymbol{v}\} \mathrm{d} A, \quad \forall \boldsymbol{v} \in \Sigma_{h}^{p} .
$$

For a given face $F \in \mathcal{F}_{h}$, we will also need the local lifting operator $\mathcal{R}_{F}:\left[L^{2}(F)\right]^{3} \rightarrow \Sigma_{h}^{p}$, defined as

$$
\left(\mathcal{R}_{F}(\boldsymbol{u}), \boldsymbol{v}\right)_{\Omega}=\int_{F} \boldsymbol{u} \cdot\{\boldsymbol{v}\} \mathrm{d} A, \quad \forall \boldsymbol{v} \in \Sigma_{h}^{p} .
$$

Note that $\mathcal{R}_{F}(\boldsymbol{u})$ vanishes outside the elements connected to the face $F$ so that for a given element $K \in \mathcal{T}_{h}$ we have the relation

$$
\mathcal{R}(\boldsymbol{u})=\sum_{F \in \mathcal{F}_{h}} \mathcal{R}_{F}(\boldsymbol{u}), \quad \forall \boldsymbol{u} \in\left[L^{2}\left(\mathcal{F}_{h}\right)\right]^{3}
$$

We also use the notation $H^{r}(\Omega)$ for the Sobolev space (with a possibly non-integer exponent).

\section{Discontinuous Galerkin Discretisation}

We now derive the DG formulation for (1). We first provide a general bilinear form where the choice of the numerical flux is not yet specified. Then we consider two different definitions of the numerical flux, each of which results in a symmetric algebraic system.

\subsection{Derivation of the Bilinear Form}

The derivation follows the same lines as the one in [28] for the Laplace operator. However, this time it is carried out for the curl-curl operator. We also refer to [2] for a unified analysis on DG methods for elliptic problems. 
We first introduce the auxiliary variable $\boldsymbol{q} \in\left[L^{2}(\Omega)\right]^{3}$ so that, instead of (1), we can consider the first-order system

$$
\begin{aligned}
\nabla \times \boldsymbol{q}-k^{2} \boldsymbol{E}=\boldsymbol{J} & \text { in } \Omega, \\
\boldsymbol{q}=\nabla \times \boldsymbol{E} & \text { in } \Omega, \\
\boldsymbol{n} \times \boldsymbol{E}=\boldsymbol{g} & \text { on } \Gamma .
\end{aligned}
$$

From here we follow the standard DG approach (given, for example, in [2] or [28] for elliptic operators): (a) multiply both equations in (9) with arbitrary test functions $\boldsymbol{\phi}, \boldsymbol{\pi} \in \Sigma_{h}^{p}$ and integrate by parts; (b) in the element boundary integrals substitute the numerical fluxes $\boldsymbol{q}_{h}^{*}$ and $\boldsymbol{E}_{h}^{*}$ for their original counterparts; (c) and finally integrate again the second equation in (9) by parts. Then we seek the pair $\left(\boldsymbol{E}_{h}, \boldsymbol{q}_{h}\right) \in \boldsymbol{\Sigma}_{h}^{p} \times \boldsymbol{\Sigma}_{h}^{p}$ such that for all test functions $(\boldsymbol{\phi}, \boldsymbol{\pi}) \in \boldsymbol{\Sigma}_{h}^{p} \times \boldsymbol{\Sigma}_{h}^{p}$ :

$$
\begin{aligned}
& \left(\boldsymbol{q}_{h}, \nabla_{h} \times \boldsymbol{\phi}\right)_{\Omega}-k^{2}\left(\boldsymbol{E}_{h}, \boldsymbol{\phi}\right)_{\Omega}+\sum_{K \in \mathcal{T}_{h}}\left(\boldsymbol{n} \times \boldsymbol{q}_{h}^{*}, \boldsymbol{\phi}\right)_{\partial K}=(\boldsymbol{J}, \boldsymbol{\phi})_{\Omega}, \\
& \left(\boldsymbol{q}_{h}, \boldsymbol{\pi}\right)_{\Omega}=\left(\nabla_{h} \times \boldsymbol{E}_{h}, \boldsymbol{\pi}\right)_{\Omega}+\sum_{K \in \mathcal{T}_{h}}\left(\boldsymbol{n} \times\left(\boldsymbol{E}_{h}^{*}-\boldsymbol{E}_{h}\right), \boldsymbol{\pi}\right)_{\partial K} .
\end{aligned}
$$

Before we proceed, we make use of the following result: for any given $\boldsymbol{u}, \boldsymbol{v} \in \Sigma_{h}^{p}$, the identity

$$
\sum_{K \in \mathcal{T}_{h}}(\boldsymbol{n} \times \boldsymbol{u}, \boldsymbol{v})_{\partial K}=-\int_{\mathcal{F}_{h}^{i}}\{\boldsymbol{u}\} \cdot \llbracket \boldsymbol{v} \rrbracket_{T} \mathrm{~d} A+\int_{\mathcal{F}_{h}^{i}}\left\{\boldsymbol{v} \rrbracket \cdot \llbracket \boldsymbol{u} \rrbracket_{T} \mathrm{~d} A+\int_{\mathcal{F}_{h}^{b}}(\boldsymbol{n} \times \boldsymbol{u}) \cdot \boldsymbol{v} \mathrm{d} A\right.
$$

holds. Combine this with (10) and (11) to obtain

$$
\begin{aligned}
\left(\boldsymbol{q}_{h},\right. & \left.\nabla_{h} \times \boldsymbol{\phi}\right)_{\Omega}-k^{2}\left(\boldsymbol{E}_{h}, \boldsymbol{\phi}\right)_{\Omega}-\int_{\mathcal{F}_{h}^{i}}\left\{\boldsymbol{q}_{h}^{*}\right\} \cdot \llbracket \boldsymbol{\phi} \rrbracket_{T} \mathrm{~d} A \\
& +\int_{\mathcal{F}_{h}^{i}}\{\boldsymbol{\phi}\} \cdot \llbracket \boldsymbol{q}_{h}^{*} \rrbracket_{T} \mathrm{~d} A+\int_{\mathcal{F}_{h}^{b}}\left(\boldsymbol{n} \times \boldsymbol{q}_{h}^{*}\right) \cdot \boldsymbol{\phi} \mathrm{d} A=(\boldsymbol{J}, \boldsymbol{\phi})_{\Omega}
\end{aligned}
$$

and

$$
\begin{aligned}
\left(\boldsymbol{q}_{h}, \boldsymbol{\pi}\right)_{\Omega}= & \left(\nabla_{h} \times \boldsymbol{E}_{h}, \boldsymbol{\pi}\right)_{\Omega}-\int_{\mathcal{F}_{h}^{i}}\left\{\boldsymbol{E}_{h}^{*}-\boldsymbol{E}_{h}\right\} \cdot \llbracket \boldsymbol{\pi} \rrbracket_{T} \mathrm{~d} A \\
& +\int_{\mathcal{F}_{h}^{i}} \llbracket \boldsymbol{\pi} \rrbracket \cdot \llbracket \boldsymbol{E}_{h}^{*}-\boldsymbol{E}_{h} \rrbracket_{T} \mathrm{~d} A+\int_{\mathcal{F}_{h}^{b}}\left(\boldsymbol{n} \times\left(\boldsymbol{E}_{h}^{*}-\boldsymbol{E}_{h}\right)\right) \cdot \boldsymbol{\pi} \mathrm{d} A .
\end{aligned}
$$

We can use the lifting operators to express - and thus eliminate-the auxiliary variable $\boldsymbol{q}_{h}$ as a function of $\boldsymbol{E}_{h}$. From (14) and from the definition of the lifting operators (5) and (6), it follows that

$$
\left.\boldsymbol{q}_{h}=\nabla_{h} \times \boldsymbol{E}_{h}-\mathcal{L}\left(\llbracket \boldsymbol{E}_{h}^{*}-\boldsymbol{E}_{h}\right\}\right)+\mathcal{R}\left(\llbracket \boldsymbol{E}_{h}^{*}-\boldsymbol{E}_{h} \rrbracket_{T}\right) .
$$

Here we have also used the boundary definition of $\mathbb{[} \cdot \rrbracket_{T}$. Substituting (15) into (13) and applying (11) results in the weak form 


$$
\begin{aligned}
\mathcal{B}\left(\boldsymbol{E}_{h}, \boldsymbol{\phi}\right):= & \left(\nabla_{h} \times \boldsymbol{E}_{h}, \nabla_{h} \times \boldsymbol{\phi}\right)_{\Omega}-k^{2}\left(\boldsymbol{E}_{h}, \boldsymbol{\phi}\right)_{\Omega} \\
& \left.-\int_{\mathcal{F}_{h}^{i}}\left\{\boldsymbol{E}_{h}^{*}-\boldsymbol{E}_{h}\right\} \cdot \llbracket \nabla_{h} \times \boldsymbol{\phi} \rrbracket_{T} \mathrm{~d} A+\int_{\mathcal{F}_{h}^{i}} \llbracket \boldsymbol{E}_{h}^{*}-\boldsymbol{E}_{h} \rrbracket_{T} \cdot \llbracket \nabla_{h} \times \boldsymbol{\phi}\right\} \mathrm{d} A \\
& -\int_{\mathcal{F}_{h}^{i}}\left\{\boldsymbol{q}_{h}^{*}\right\} \cdot \llbracket \boldsymbol{\phi} \rrbracket_{T} \mathrm{~d} A+\int_{\mathcal{F}_{h}^{i}} \llbracket \boldsymbol{q}_{h}^{*} \rrbracket_{T} \cdot\{\boldsymbol{\phi}\} \mathrm{d} A \\
& +\int_{\mathcal{F}_{h}^{b}}\left(\boldsymbol{n} \times\left(\boldsymbol{E}_{h}^{*}-\boldsymbol{E}_{h}\right)\right) \cdot\left(\nabla_{h} \times \boldsymbol{\phi}\right) \mathrm{d} A-\int_{\mathcal{F}_{h}^{b}} \boldsymbol{q}_{h}^{*} \cdot(\boldsymbol{n} \times \boldsymbol{\phi}) \mathrm{d} A=(\boldsymbol{J}, \boldsymbol{\phi})_{\Omega} .
\end{aligned}
$$

This is the general primal formulation where one still has freedom to make choices about the numerical fluxes $\boldsymbol{E}_{h}^{*}$ and $\boldsymbol{q}_{h}^{*}$ that are most suitable for the problem. An overview of different fluxes for the Poisson equation is given in [2].

\subsection{Numerical Fluxes}

At this point, we specify the numerical fluxes $\boldsymbol{E}_{h}^{*}$ and $\boldsymbol{q}_{h}^{*}$ in (16). We investigate two different formulations, one of which results in the IP-DG formulation that was thoroughly analysed in [18]. The other is similar to the stabilised central flux, except that in the stabilisation term we use the local lifting operator (7). Note that in both cases the numerical fluxes are consistent, i.e. $\forall \boldsymbol{E}, \boldsymbol{q} \in H(\operatorname{curl}, \Omega)$ the relations $\{\boldsymbol{E}\}_{T}=\boldsymbol{n} \times \boldsymbol{E},\{\boldsymbol{q}\}=\boldsymbol{n} \times \boldsymbol{q}_{h}, \llbracket \boldsymbol{E} \rrbracket_{T}=\mathbf{0}$ and $\llbracket \boldsymbol{q} \rrbracket_{T}=\mathbf{0}$ hold.

\subsubsection{Interior-Penalty Flux}

First, we define the numerical fluxes so that they correspond to the IP flux,

$$
\begin{array}{lll}
\boldsymbol{E}_{h}^{*}=\left\{\boldsymbol{E}_{h}\right\}, & \boldsymbol{q}_{h}^{*}=\left\{\nabla_{h} \times \boldsymbol{E}_{h}\right\}-\mathrm{a}_{F} \llbracket \boldsymbol{E}_{h} \rrbracket_{T}, & \text { if } F \in \mathcal{F}_{h}^{i}, \\
\boldsymbol{n} \times \boldsymbol{E}_{h}^{*}=\boldsymbol{g}, & \boldsymbol{q}_{h}^{*}=\nabla_{h} \times \boldsymbol{E}_{h}-\mathrm{a}_{F}\left(\boldsymbol{n} \times \boldsymbol{E}_{h}\right)+\mathrm{a}_{F} \boldsymbol{g}, & \text { if } F \in \mathcal{F}_{h}^{b},
\end{array}
$$

with $\mathrm{a}_{F}$ being the penalty parameter. We can now transform the following face integrals as

$$
\begin{aligned}
\int_{\mathcal{F}_{h}^{i}} \llbracket \boldsymbol{E}_{h}^{*}-\boldsymbol{E}_{h} \rrbracket_{T} \cdot\left\{\nabla_{h} \times \boldsymbol{\phi}\right\} \mathrm{d} A=-\int_{\mathcal{F}_{h}^{i}} \llbracket \boldsymbol{E}_{h} \rrbracket_{T} \cdot\left\{\nabla_{h} \times \boldsymbol{\phi}\right\} \mathrm{d} A, \\
\int_{\mathcal{F}_{h}^{b}}\left(\boldsymbol{n} \times\left(\boldsymbol{E}_{h}^{*}-\boldsymbol{E}_{h}\right)\right) \cdot\left(\nabla_{h} \times \boldsymbol{\phi}\right) \mathrm{d} A=\int_{\mathcal{F}_{h}^{b}}\left(\boldsymbol{g}-\boldsymbol{n} \times \boldsymbol{E}_{h}\right) \cdot\left(\nabla_{h} \times \boldsymbol{\phi}\right) \mathrm{d} A, \\
\left.\int_{\mathcal{F}_{h}^{i}}\left\{\boldsymbol{q}_{h}^{*}\right\} \cdot \llbracket \boldsymbol{\phi} \rrbracket_{T} \mathrm{~d} A=\int_{\mathcal{F}_{h}^{i}} \llbracket \nabla_{h} \times \boldsymbol{E}_{h}\right\} \cdot \llbracket \boldsymbol{\phi} \rrbracket_{T} \mathrm{~d} A-\int_{\mathcal{F}_{h}^{i}} \mathrm{a}_{F} \llbracket \boldsymbol{E}_{h} \rrbracket_{T} \cdot \llbracket \boldsymbol{\phi} \rrbracket_{T} \mathrm{~d} A, \\
\int_{\mathcal{F}_{h}^{b}}\left(\boldsymbol{n} \times \boldsymbol{q}_{h}^{*}\right) \cdot \boldsymbol{\phi} \mathrm{d} A=-\int_{\mathcal{F}_{h}^{b}}\left(\nabla_{h} \times \boldsymbol{E}_{h}\right) \cdot(\boldsymbol{n} \times \boldsymbol{\phi}) \mathrm{d} A \\
+\int_{\mathcal{F}_{h}^{b}} \mathrm{a}_{F}\left(\boldsymbol{n} \times \boldsymbol{E}_{h}\right) \cdot(\boldsymbol{n} \times \boldsymbol{\phi}) \mathrm{d} A-\int_{\mathcal{F}_{h}^{b}} \mathrm{a}_{F} \boldsymbol{g} \cdot(\boldsymbol{n} \times \boldsymbol{\phi}) \mathrm{d} A,
\end{aligned}
$$

while the other face integrals are zero. If we plug these back to (16), define the bilinear form $\mathcal{B}_{h}^{i p}: \Sigma_{h}^{p} \times \Sigma_{h}^{p} \rightarrow \mathbb{R}$ as 


$$
\begin{aligned}
\mathcal{B}_{h}^{i p}\left(\boldsymbol{E}_{h}, \boldsymbol{\phi}\right):= & \left(\nabla_{h} \times \boldsymbol{E}_{h}, \nabla_{h} \times \boldsymbol{\phi}\right)_{\Omega}-k^{2}\left(\boldsymbol{E}_{h}, \boldsymbol{\phi}\right)_{\Omega}-\int_{\mathcal{F}_{h}} \llbracket \boldsymbol{E}_{h} \rrbracket_{T} \cdot\left\{\nabla_{h} \times \boldsymbol{\phi}\right\} \mathrm{d} A \\
& -\int_{\mathcal{F}_{h}}\left\{\nabla_{h} \times \boldsymbol{E}_{h}\right\} \cdot \llbracket \boldsymbol{\phi} \rrbracket_{T} \mathrm{~d} A+\int_{\mathcal{F}_{h}} \mathrm{a}_{F} \llbracket \boldsymbol{E} \rrbracket_{T} \cdot \llbracket \boldsymbol{\phi} \rrbracket_{T} \mathrm{~d} A
\end{aligned}
$$

and the linear form $\mathcal{J}_{h}^{i p}: \Sigma_{h}^{p} \rightarrow \mathbb{R}$ as

$$
\mathcal{J}_{h}^{i p}(\boldsymbol{\phi}):=(\boldsymbol{J}, \boldsymbol{\phi})_{\Omega}-\int_{\mathcal{F}_{h}^{b}} \boldsymbol{g} \cdot\left(\nabla_{h} \times \boldsymbol{\phi}\right) \mathrm{d} A+\int_{\mathcal{F}_{h}^{b}} a_{F} \boldsymbol{g} \cdot(\boldsymbol{n} \times \boldsymbol{\phi}) \mathrm{d} A,
$$

we have the IP-DG method for the time-harmonic Maxwell equations, formulated as follows. Find $\boldsymbol{E}_{h} \in \Sigma_{h}^{p}$ such that for all $\boldsymbol{\phi} \in \Sigma_{h}^{p}$ the relation

$$
\mathcal{B}_{h}^{i p}\left(\boldsymbol{E}_{h}, \boldsymbol{\phi}\right)=\mathcal{J}_{h}^{i p}(\boldsymbol{\phi})
$$

is satisfied. Note that in (18) we no longer distinguish explicitly between internal and boundary faces. This is permissible thanks to the definitions of the average and the tangential jump at the boundary.

\subsubsection{Numerical Flux of Brezzi Formulation}

As a next step, we define the numerical fluxes in the manner of Brezzi et al. [8]:

$$
\begin{array}{lll}
\boldsymbol{E}_{h}^{*}=\left\{\boldsymbol{E}_{h}\right\}, & \boldsymbol{q}_{h}^{*}=\left\{\boldsymbol{q}_{h}\right\}-\alpha_{\mathcal{R}}\left(\llbracket \boldsymbol{E}_{h} \rrbracket_{T}\right), & \text { if } F \in \mathcal{F}_{h}^{i}, \\
\boldsymbol{n} \times \boldsymbol{E}_{h}^{*}=\boldsymbol{g}, & \boldsymbol{q}_{h}^{*}=\boldsymbol{q}_{h}-\alpha_{\mathcal{R}}\left(\boldsymbol{n} \times \boldsymbol{E}_{h}\right)+\alpha_{\mathcal{R}}(\boldsymbol{g}), & \text { if } F \in \mathcal{F}_{h}^{b},
\end{array}
$$

where $\alpha_{\mathcal{R}}(\boldsymbol{u})=\eta_{F}\left\{\mathcal{R}_{F}\left(\boldsymbol{u}_{h}\right)\right\}$ for $F \in \mathcal{F}_{h}$ and $\eta_{F} \in \mathbb{R}$. Following the same line of argument as before and using (15), the bilinear form (16) now transforms as

$$
\begin{aligned}
\mathcal{B}\left(\boldsymbol{E}_{h}, \boldsymbol{\phi}\right):= & \left(\nabla_{h} \times \boldsymbol{E}_{h}, \nabla_{h} \times \boldsymbol{\phi}\right)_{\Omega}-k^{2}\left(\boldsymbol{E}_{h}, \boldsymbol{\phi}\right)_{\Omega} \\
& \left.-\int_{\mathcal{F}_{h}} \llbracket \boldsymbol{E}_{h} \rrbracket_{T} \cdot \llbracket \nabla_{h} \times \boldsymbol{\phi}\right\} \mathrm{d} A-\int_{\mathcal{F}_{h}}\left\{\nabla_{h} \times \boldsymbol{E}_{h}\right\} \cdot \llbracket \boldsymbol{\phi} \rrbracket_{T} \mathrm{~d} A \\
& -\int_{\mathcal{F}_{h}}\left\{\mathcal{R}\left(\llbracket \boldsymbol{E}_{h}^{*}-\boldsymbol{E}_{h} \rrbracket_{T}\right)\right\} \cdot \llbracket \boldsymbol{\phi} \rrbracket_{T} \mathrm{~d} A+\sum_{F \in \mathcal{F}_{h}} \int_{F} \eta_{F}\left\{\mathcal{R}_{F}\left(\llbracket \boldsymbol{E}_{h} \rrbracket_{T}\right)\right\} \cdot \llbracket \boldsymbol{\phi} \rrbracket_{T} \mathrm{~d} A \\
& +\int_{\mathcal{F}_{h}^{b}} \boldsymbol{g} \cdot\left(\nabla_{h} \times \boldsymbol{\phi}\right) \mathrm{d} A-\sum_{F \in \mathcal{F}_{h}^{b}} \int_{F} \eta_{F} \mathcal{R}_{F}(\boldsymbol{g}) \cdot(\boldsymbol{n} \times \boldsymbol{\phi}) \mathrm{d} A .
\end{aligned}
$$

We can now use the relation

$$
\begin{aligned}
\int_{\mathcal{F}_{h}} & \left\{\mathcal{R}\left(\llbracket \boldsymbol{E}_{h}^{*}-\boldsymbol{E}_{h} \rrbracket_{T}\right)\right\} \cdot \llbracket \boldsymbol{\phi} \rrbracket_{T} \mathrm{~d} A \\
= & \left(\mathcal{R}\left(\llbracket \boldsymbol{E}_{h}^{*}-\boldsymbol{E}_{h} \rrbracket_{T}\right), \mathcal{R}\left(\llbracket \boldsymbol{\phi} \rrbracket_{T}\right)\right)_{\Omega} \\
\approx & n_{f} \sum_{F \in \mathcal{F}_{h}}\left(\mathcal{R}_{F}\left(\llbracket \boldsymbol{E}_{h}^{*}-\boldsymbol{E}_{h} \rrbracket_{T}\right), \mathcal{R}_{F}\left(\llbracket \boldsymbol{\phi} \rrbracket_{T}\right)\right)_{\Omega}
\end{aligned}
$$




$$
\begin{aligned}
& =-n_{f} \sum_{F \in \mathcal{F}_{h}^{i}}\left(\mathcal{R}_{F}\left(\llbracket \boldsymbol{E}_{h} \rrbracket_{T}\right), \mathcal{R}_{F}\left(\llbracket \boldsymbol{\phi} \rrbracket_{T}\right)\right)_{\Omega}+n_{f} \sum_{F \in \mathcal{F}_{h}^{b}}\left(\mathcal{R}_{F}\left(\boldsymbol{g}-\llbracket \boldsymbol{E}_{h} \rrbracket_{T}\right), \mathcal{R}_{F}\left(\llbracket \boldsymbol{\phi} \rrbracket_{T}\right)\right)_{\Omega} \\
& =-n_{f} \sum_{F \in \mathcal{F}_{h}}\left(\mathcal{R}_{F}\left(\llbracket \boldsymbol{E}_{h} \rrbracket_{T}\right), \mathcal{R}_{F}\left(\llbracket \boldsymbol{\phi} \rrbracket_{T}\right)\right)_{\Omega}+n_{f} \sum_{F \in \mathcal{F}_{h}^{b}}\left(\mathcal{R}_{F}(\boldsymbol{g}), \mathcal{R}_{F}\left(\llbracket \boldsymbol{\phi} \rrbracket_{T}\right)\right)_{\Omega},
\end{aligned}
$$

where $n_{f}$ is the number of faces of an element.

Let us introduce the bilinear form $\mathcal{B}_{h}^{b r}: \Sigma_{h}^{p} \times \Sigma_{h}^{p} \rightarrow \mathbb{R}$ and the linear form $\mathcal{J}_{h}^{b r}: \Sigma_{h}^{p} \rightarrow \mathbb{R}$ as

$$
\begin{aligned}
\mathcal{B}_{h}^{b r}\left(\boldsymbol{E}_{h}, \boldsymbol{\phi}\right)= & \left(\nabla_{h} \times \boldsymbol{E}_{h}, \nabla_{h} \times \boldsymbol{\phi}\right)_{\Omega}-k^{2}\left(\boldsymbol{E}_{h}, \boldsymbol{\phi}\right)_{\Omega} \\
& -\int_{\mathcal{F}_{h}} \llbracket \boldsymbol{E}_{h} \rrbracket_{T} \cdot\left\{\nabla_{h} \times \boldsymbol{\phi}\right\} \mathrm{d} A-\int_{\mathcal{F}_{h}}\left\{\nabla_{h} \times \boldsymbol{E}_{h}\right\} \cdot \llbracket \boldsymbol{\phi} \rrbracket_{T} \mathrm{~d} A \\
& +\sum_{F \in \mathcal{F}_{h}}\left(\eta_{F}+n_{f}\right)\left(\mathcal{R}_{F}\left(\llbracket \boldsymbol{E} \rrbracket_{T}\right), \mathcal{R}_{F}\left(\llbracket \boldsymbol{\phi} \rrbracket_{T}\right)\right)_{\Omega},
\end{aligned}
$$

and

$$
\mathcal{J}_{h}^{b r}(\boldsymbol{\phi})=(\boldsymbol{J}, \boldsymbol{\phi})_{\Omega}-\int_{\mathcal{F}_{h}^{b}} \boldsymbol{g} \cdot\left(\nabla_{h} \times \boldsymbol{\phi}\right) \mathrm{d} A+\sum_{F \in \mathcal{F}_{h}^{b}}\left(\eta_{F}+n_{f}\right)\left(\mathcal{R}_{F}(\boldsymbol{g}), \mathcal{R}_{F}(\boldsymbol{n} \times \boldsymbol{\phi})\right)_{\Omega},
$$

respectively, then the discrete formulation for the time-harmonic Maxwell equations can be written as follows. Find $\boldsymbol{E}_{h} \in \Sigma_{h}^{p}$ such that for all $\boldsymbol{\phi} \in \Sigma_{h}^{p}$ the relation

$$
\mathcal{B}_{h}^{b r}\left(\boldsymbol{E}_{h}, \boldsymbol{\phi}\right)=\mathcal{J}_{h}^{b r}(\boldsymbol{\phi})
$$

is satisfied.

The discrete counterparts of the eigenvalue problem (2) for the IP and Brezzi type DG methods naturally follow from (20) and (25), i.e. find $k^{2} \in \mathbb{R}_{0}^{+}$such that for some $\boldsymbol{E}_{h} \in \Sigma_{h}^{p}$, respectively, $B_{h}^{i p}\left(\boldsymbol{E}_{h}, \boldsymbol{\phi}\right)=0$ and $B_{h}^{b r}\left(\boldsymbol{E}_{h}, \boldsymbol{\phi}\right)=0$ are satisfied for all $\boldsymbol{\phi} \in \Sigma_{h}^{p}$.

\section{Explicit Parameter and Error Estimates}

Both the IP and the Brezzi type DG formulations, given respectively by (20) and (25), contain parameters that need to be set to ensure stability. In this section, we provide explicit formulations for these parameters. First, we present an accurate lower bound for the lifting operator $\mathcal{R}_{F}$ on tetrahedral elements, extending the proof in [25] for hexahedra. Next, we recall the statements in [18], which are necessary for the convergence proof and keep track of all constant terms. Using these results we provide optimal penalty parameter for both the IP and the Brezzi type DG method. We also point out that these conditions are sufficient for a spurious-free convergence for the associated eigenvalue problems, discussed in [9].

In the consecutive estimates $K^{L}$ and $K^{R}$ denote the adjacent elements to the face $F \in \mathcal{F}_{h}$ and we introduce

$$
M_{F}=\max \left\{\frac{S(F)}{V\left(K^{L}\right)}, \frac{S(F)}{V\left(K^{R}\right)}\right\},
$$

where $S$ and $V$ denote the surface and volume, respectively. 


\subsection{Bounds for the Lifting Operator}

Lemma 1 For an arbitrary face $F_{K}$ of $K \in \mathcal{T}_{h}$ any $v \in \Sigma_{h}^{p}$ satisfies the inequality

$$
\frac{2}{3} \frac{p^{2}}{F^{2}(p)} \frac{S\left(F_{K}\right)}{V(K)}\left\|\llbracket v \rrbracket_{T}\right\|_{0, F_{K}}^{2} \leq\left\|\mathcal{R}_{F}\left(\llbracket v \rrbracket_{T}\right)\right\|_{K}^{2},
$$

where $F^{2}(p)=8 \sum_{i=\frac{p}{2}}^{p} \frac{1}{2 i+3}$ if $p$ is even and $F^{2}(p)=\frac{8 p^{2}}{(p+1)^{2}} \sum_{i=\frac{p-1}{2}+1}^{p} \frac{1}{2 i+3}$ if $p$ is odd.

Proof The proof is divided into three steps.

Step 1: Extension operator on the reference tetrahedron. We first consider a reference tetrahedron $\hat{K}$ with vertices $(1,1,1),(-1,1,1),(1,-1,1),(1,1,-1)$ and define an extension operator corresponding to the face $\hat{F}$ opposite to $(1,1,1)$. Let $\Delta_{s}$ denote a triangle with vertices $(s, 1,1),(1, s, 1),(1,1, s)$. An arbitrary point $(\xi, \eta, \zeta)$ can be represented as

$$
(\xi, \eta, \zeta)=(1, s, 1)+u(0,1-s, s-1)+v(s-1,1-s, 0),
$$

where $0 \leq u, v, u+v \leq 1$ and $-1 \leq s \leq 1$, hence $\hat{F}=\Delta_{-1}$. The Jacobian of the mapping $(\xi, \eta, \zeta) \rightarrow(u, v, s)$ is

$$
\left(\begin{array}{ccc}
0 & s-1 & v \\
1-s & 1-s & 1-u-v \\
s-1 & 0 & u
\end{array}\right)
$$

with the determinant $(1-s)^{2}$ and under this transformation the face $\hat{F}$ is mapped to the face $\tilde{F}$.

We now define the extension of the polynomial $\tilde{\phi}: \tilde{F} \rightarrow \mathbb{R}$, which is given in terms of the local coordinates $(u, v)$. Note that the transformation $(\xi, \eta, \zeta) \rightarrow(u, v, s)$ is linear from $\hat{F}$ to $\tilde{F}$ and therefore

$$
\int_{\tilde{F}}|\tilde{\phi}|^{2}=\frac{S(\tilde{F})}{S(\hat{F})} \int_{\hat{F}}|\hat{\phi}|^{2}=\frac{1}{4 \sqrt{3}} \int_{\hat{F}}|\hat{\phi}|^{2}
$$

If the order $p$ of the polynomial $\tilde{\phi}$ is even, the extension $\hat{E}(\tilde{\phi})$ is defined as

$$
\hat{E}(\tilde{\phi})(u, v, s)=\frac{2}{p} \sum_{j=\frac{p}{2}+1}^{p} P_{j}^{(0,2)}(-s) \tilde{\phi}(u, v),
$$

where $P_{j}^{(0,2)}$ denotes the $j$ th-order Jacobi polynomial on $(-1,1)$ with the weight function $w(x)=(1+x)^{2}$ and $P_{j}^{(0,2)}(1)=1$. It is also known that

$$
\int_{-1}^{1}(1+x)^{2} P_{i}^{(0,2)}(x) P_{j}^{(0,2)}(x) \mathrm{d} x=\frac{2^{3} \cdot \Gamma(j+3) \Gamma(j+1)}{j ! \cdot(2 j+3) \Gamma(j+3)} 8 \delta_{i j}=\frac{8 \delta_{i j}}{2 j+3} .
$$

The identity in (30) gives that $\hat{E}(\tilde{\phi})(u, v,-1)=\tilde{\phi}(u, v)$. In terms of $\xi, \eta, \zeta$, we have, using (27) with $\tilde{\phi}(u, v)=\hat{\phi}(\xi, \eta)$ that

$$
\hat{E}(\hat{\phi})(\xi, \eta, \zeta)=\hat{\phi}(\xi, \eta) \quad \text { at } \hat{F},
$$


hence $\hat{E}(\hat{\phi})$ is in fact an extension of $\hat{\phi}$. Using (28), (30) and (29), we have

$$
\begin{aligned}
& \int_{\hat{K}}|\hat{E}(\hat{\phi})(\xi, \eta, \zeta)|^{2} \\
& =\int_{-1}^{1} \int_{0}^{1} \int_{0}^{1-v}|\hat{E}(\tilde{\phi})(u, v, s)|^{2}(1-s)^{2} \mathrm{~d} u \mathrm{~d} v \mathrm{~d} s \\
& =\int_{-1}^{1} \int_{0}^{1} \int_{0}^{1-v}\left|\frac{2}{p} \sum_{i=\frac{p}{2}+1}^{p} P_{i}^{(0,2)}(-s) \tilde{\phi}(u, v)\right|^{2}(1-s)^{2} \mathrm{~d} u \mathrm{~d} v \mathrm{~d} s \\
& =\frac{4}{p^{2}} \int_{-1}^{1} \sum_{i=\frac{p}{2}+1}^{p} \sum_{j=\frac{p}{2}+1}^{p} P_{i}^{(0,2)}(-s) P_{j}^{(0,2)}(-s)(1-s)^{2} \mathrm{~d} s \int_{0}^{1} \int_{0}^{1-v}|\tilde{\phi}(u, v)|^{2} \mathrm{~d} u \mathrm{~d} v \\
& =\frac{4}{p^{2}} \sum_{i=\frac{p}{2}+1}^{p} \frac{8}{2 i+3} \int_{\tilde{F}}|\tilde{\phi}|^{2}=\frac{1}{p^{2}} \sum_{i=\frac{p}{2}+1}^{p} \frac{8}{\sqrt{3}} \frac{1}{2 i+3} \int_{\hat{F}}|\hat{\phi}|^{2}
\end{aligned}
$$

As a result, we obtain the relation

$$
\|\hat{E}(\hat{\phi})\|_{0, \hat{K}}=\frac{1}{\sqrt[4]{3}} \frac{1}{p} F(p)\|\hat{\phi}\|_{0, \hat{F}},
$$

where

$$
F^{2}(p)=8 \sum_{i=\frac{p}{2}+1}^{p} \frac{1}{2 i+3} \text { if } p \text { is even. }
$$

Analogously, for odd $p$ we define the extension as

$$
\hat{E}(\tilde{\phi})(u, v, s)=\frac{2}{p+1} \sum_{i=\frac{p-1}{2}+1}^{p} P_{i}^{(0,2)}(-s) \tilde{\phi}(u, v)
$$

and the same derivation as in (31) gives that

$$
\|\hat{E}(\hat{\phi})\|_{0, \hat{K}}^{2}=\frac{1}{\sqrt{3}(p+1)^{2}} \sum_{i=\frac{p-1}{2}+1}^{p} \frac{8}{2 i+3} \int_{\hat{F}}|\hat{\phi}|^{2}=\frac{1}{\sqrt{3}} \frac{F^{2}(p)}{p^{2}}\|\hat{\phi}\|_{0, \hat{F}}^{2},
$$

such that we have

$$
F^{2}(p)=\frac{8 p^{2}}{(p+1)^{2}} \sum_{i=\frac{p-1}{2}+1}^{p} \frac{1}{2 i+3} \quad \text { if } p \text { is odd }
$$

For computing the norm of the extension operator $\hat{E}$, both for odd and even $p$, we use the estimates

$$
\sum_{i=\frac{p}{2}+1}^{p} \frac{1}{2 i+3} \leq \int_{\frac{p}{2}}^{p} \frac{1}{2 t+3} \mathrm{~d} t=\frac{1}{2} \ln \left(\frac{2 p+3}{p+3}\right) \leq \frac{1}{2} \ln 2
$$


and

$$
\sum_{i=\frac{p-1}{2}+1}^{p} \frac{1}{2 i+3} \leq \int_{\frac{p-1}{2}}^{p} \frac{1}{2 t+3} \mathrm{~d} t=\frac{1}{2} \ln \left(\frac{2 p+3}{p+2}\right) \leq \frac{1}{2} \ln 2
$$

and obtain the simple estimate

$$
F^{2}(p) \leq 4 \ln 2 .
$$

The estimate in (36) is sharp as $\lim p \rightarrow \infty$.

Step 2: Extension operator on a general tetrahedron. For an arbitrary tetrahedron $K$ with a face $F_{K}$ we define the affine transformation $T_{K}: \hat{K} \rightarrow K$ as

$$
T_{K}(\hat{\boldsymbol{x}})=J_{K} \hat{\boldsymbol{x}}+\boldsymbol{b}, \quad \text { where } \boldsymbol{b} \in \mathbb{R}^{3}, J_{K} \in \mathbb{R}^{3 \times 3} \text { and } T_{K}(\hat{F})=F_{K} .
$$

The extension $E$ of a function $\phi: F_{K} \rightarrow \mathbb{R}$ is given then as follows.

- We define the function $\hat{\phi}: \hat{F} \rightarrow \mathbb{R}$ with

$$
\hat{\phi}(\hat{\boldsymbol{x}}):=\phi\left(T_{K} \hat{\boldsymbol{x}}\right)
$$

- We extend $\hat{\phi}$ to $\hat{E}(\hat{\phi})$ using the method in Step 1 .

- The extension to $K$ is given by

$$
E(\phi)(\boldsymbol{x}):=\hat{E}(\hat{\phi})\left(T_{K}^{-1} \boldsymbol{x}\right) .
$$

As $J_{K}$ is linear, we can apply a simple change of variables $\boldsymbol{x}=T_{K}(\hat{\boldsymbol{x}})$ for computing the integral of any $g \in L_{1}(K)$ :

$$
\int_{K} g(\boldsymbol{x}) \mathrm{d} \boldsymbol{x}=\left|\operatorname{det} J_{K}\right| \int_{\hat{K}} \hat{g}(\hat{\boldsymbol{x}}) \mathrm{d} \hat{\boldsymbol{x}}=\frac{V(K)}{V(\hat{K})} \int_{\hat{K}} \hat{g}(\hat{\boldsymbol{x}}) \mathrm{d} \hat{\boldsymbol{x}} .
$$

Since the restriction of $J_{K}$ to the face $F_{K}$ of $K$ remains affine, we also have, as in (29), that

$$
\int_{F_{K}} g(\boldsymbol{x}) \mathrm{d} \boldsymbol{x}=\frac{S\left(F_{K}\right)}{S(\hat{F})} \int_{\hat{F}} \hat{g}(\hat{\boldsymbol{x}}) \mathrm{d} \hat{\boldsymbol{x}} .
$$

Using (37) with the relations (32), (34) and (38) we obtain

$$
\begin{aligned}
\|E(\phi)\|_{0, K}^{2} & =\frac{V(K)}{V(\hat{K})}\|\hat{E}(\hat{\phi})\|_{0, \hat{K}}^{2}=\frac{V(K)}{V(\hat{K})} \frac{1}{\sqrt{3}} \frac{F^{2}(p)}{p^{2}}\|\hat{\phi}\|_{0, \hat{F}}^{2} \\
& =\frac{V(K)}{V(\hat{K})} \frac{1}{\sqrt{3}} \frac{S(\hat{F})}{S\left(F_{K}\right)} \frac{F^{2}(p)}{p^{2}}\|\phi\|_{0, F_{K}}^{2}=\frac{S(\hat{F})}{V(\hat{K})} \frac{V(K)}{S\left(F_{K}\right)} \frac{1}{\sqrt{3}} \frac{F^{2}(p)}{p^{2}}\|\phi\|_{0, F_{K}}^{2} .
\end{aligned}
$$

On the reference tetrahedron $\hat{K}$ we extended $\hat{\phi}$ from the face $\hat{F}$ with $S(\hat{F})=2 \sqrt{3}$ and we have $V(\hat{K})=\frac{4}{3}$, therefore (39) reduces to

$$
\|E(\phi)\|_{0, K}^{2}=\frac{3}{2} \frac{V(K)}{S\left(F_{K}\right)} \frac{F^{2}(p)}{p^{2}}\|\phi\|_{0, F_{K}}^{2} .
$$


Step 3: The inequality for the jump term. Using the estimate in (40), the definition of $\mathcal{R}_{F}$ in (7) with the fact that $E\left(\llbracket v \rrbracket_{T}\right)$ is continuous on $\partial K$ we obtain

$$
\begin{aligned}
\left\|\llbracket v \rrbracket_{T}\right\|_{0, F_{K}}^{2} & =\int_{F} \llbracket v \rrbracket_{T} \cdot E\left(\llbracket v \rrbracket_{T}\right)=\int_{K} \mathcal{R}_{F}\left(\llbracket v \rrbracket_{T}\right) \cdot E\left(\llbracket v \rrbracket_{T}\right) \\
& \leq\left\|\mathcal{R}_{F}\left(\llbracket v \rrbracket_{T}\right)\right\|_{0, K}\left(\frac{3}{2} \frac{V(K)}{S\left(F_{K}\right)} \frac{F^{2}(p)}{p^{2}}\right)^{\frac{1}{2}}\left\|\llbracket v \rrbracket_{T}\right\|_{0, F_{K}},
\end{aligned}
$$

which gives the desired inequality.

Remark Since $K$ is an arbitrary element adjacent to $F_{K}$, we can rewrite the estimate in Lemma 1 as

$$
\frac{2}{3} M_{F} \frac{p^{2}}{F^{2}(p)}\left\|\llbracket v \rrbracket_{T}\right\|_{0, F}^{2} \leq\left\|\mathcal{R}_{F} \llbracket v \rrbracket_{T}\right\|_{0, K}^{2} .
$$

In the following lemma, we will make use of the inverse trace inequality on an arbitrary face $F$ of the element $K$

$$
\|\boldsymbol{w}\|_{0, F}^{2} \leq \frac{(p+1)(p+3)}{3} \frac{S(F)}{V(K)}\|\boldsymbol{w}\|_{0, K}^{2}
$$

in $\Sigma_{h}^{p}$, which is proved in Theorem 4 in [30].

Lemma 2 For every face $F \in \mathcal{F}_{h}$ and every $v \in \Sigma_{h}^{p}$ we have the inequality

$$
\left\|\mathcal{R}_{F}\left(\llbracket v \rrbracket_{T}\right)\right\|_{0} \leq \sqrt{\frac{M_{F}(p+1)(p+3)}{6}}\left\|\llbracket v \rrbracket_{T}\right\|_{0, F} .
$$

Proof The definition of the $\left[L_{2}(\Omega)\right]^{3}$ norm and the trace inequality in (42) give that for an arbitrary $\boldsymbol{v} \in \Sigma_{h}^{p}$

$$
\begin{aligned}
& \left\|\mathcal{R}_{F}\left(\llbracket \boldsymbol{v} \rrbracket_{T}\right)\right\|_{0} \\
& =\sup _{\boldsymbol{w} \in \Sigma_{h}^{p}} \frac{\int_{\Omega} \mathcal{R}_{F}\left(\llbracket \boldsymbol{v} \rrbracket_{T}\right) \cdot \boldsymbol{w}}{\|\boldsymbol{w}\|_{0}}=\sup _{\boldsymbol{w} \in \Sigma_{h}^{p}} \frac{\left.\int_{F} \llbracket \boldsymbol{v} \rrbracket_{T} \cdot \llbracket \boldsymbol{w}\right\}}{\|\boldsymbol{w}\|_{0}} \\
& \leq \sup _{\boldsymbol{w} \in \Sigma_{h}^{p}} \frac{\left\|\llbracket \boldsymbol{v} \rrbracket_{T}\right\|_{0, F}\left(\int_{F}\left(\frac{\left.\boldsymbol{w}\right|_{\partial K^{L}}+\left.\boldsymbol{w}\right|_{\partial K^{R}}}{2}\right)^{2}\right)^{\frac{1}{2}}}{\|\boldsymbol{w}\|_{0}} \\
& \leq \sup _{\boldsymbol{w} \in \Sigma_{h}^{p}} \frac{\left\|\llbracket \boldsymbol{v} \rrbracket_{T}\right\|_{0, F}\left(\frac{1}{2}\left(\|\boldsymbol{w}\|_{\partial K^{L}}^{2}+\|\boldsymbol{w}\|_{\partial K^{R}}^{2}\right)\right)^{\frac{1}{2}}}{\|\boldsymbol{w}\|_{0}} \\
& \leq \sup _{\boldsymbol{w} \in \Sigma_{h}^{p}} \frac{\sqrt{M_{F} \frac{(p+1)(p+3)}{3}\left\|\llbracket \boldsymbol{v} \rrbracket_{T}\right\|_{0, F}}}{\|\boldsymbol{w}\|_{0}} \\
& \quad \times\left(\frac{1}{2}\left(\frac{V\left(K^{L}\right)}{S(F)} \frac{3}{(p+1)(p+3)}\|\boldsymbol{w}\|_{\partial K^{L}}^{2}+\frac{V\left(K^{R}\right)}{S(F)} \frac{3}{(p+1)(p+3)}\|\boldsymbol{w}\|_{\partial K^{R}}^{2}\right)\right)^{\frac{1}{2}} \\
& \\
& \quad
\end{aligned}
$$




$$
\begin{aligned}
& \leq \sup _{\boldsymbol{w} \in \Sigma_{h}^{p}} \frac{\sqrt{M_{F} \frac{(p+1)(p+3)}{3}}\left\|\llbracket \boldsymbol{v} \rrbracket_{T}\right\|_{0, F}\left(\frac{1}{2}\left(\|\boldsymbol{w}\|_{0, K^{L}}^{2}+\|\boldsymbol{w}\|_{0, K^{R}}^{2}\right)\right)^{\frac{1}{2}}}{\|\boldsymbol{w}\|_{0}} \\
& \leq \sup _{\boldsymbol{w} \in \Sigma_{h}^{p}} \frac{\sqrt{M_{F} \frac{(p+1)(p+3)}{6}}\left\|\llbracket \boldsymbol{v} \rrbracket_{T}\right\|_{0, F}\|\boldsymbol{w}\|_{0}}{\|\boldsymbol{w}\|_{0}}=\sqrt{\frac{M_{F}(p+1)(p+3)}{6}}\left\|\llbracket \boldsymbol{v} \rrbracket_{T}\right\|_{0, F},
\end{aligned}
$$

as stated.

\subsection{Gårding Inequalities and Continuity Estimates}

We begin by proving the Gårding inequality for the bilinear form of the Brezzi type DG formulation (25).

Lemma 3 There exist constants $\left\{\eta_{F, 0}\right\}_{F \in \mathcal{F}_{h}}$, independent of the discretisation parameter $h=\max _{K \in \mathcal{T}_{h}} \operatorname{diam} K$ and the wave number $k$, such that for all $\boldsymbol{v} \in \Sigma_{h}^{p}$ and all parameters $\eta_{F} \geq \eta_{F, 0}$ we have the following inequality

$$
\mathcal{B}_{h}^{b r}(\boldsymbol{v}, \boldsymbol{v}) \geq \beta^{2}\|\boldsymbol{v}\|_{\mathrm{DG}}^{2}-\left(k^{2}+\beta^{2}\right)\|\boldsymbol{v}\|_{0}^{2} .
$$

Proof The right hand side of (44) can be rewritten as

$$
\beta^{2}\left(\left\|\nabla_{h} \times \boldsymbol{v}\right\|_{0}^{2}+\left\|\mathrm{h}^{-\frac{1}{2}} \llbracket \boldsymbol{v} \rrbracket_{T}\right\|_{0, \mathcal{F}_{h}}^{2}\right)-k^{2}\|\boldsymbol{v}\|_{0}^{2}
$$

Therefore, using (23) it is sufficient to prove that

$$
\begin{aligned}
& \left\|\nabla_{h} \times \boldsymbol{v}\right\|_{0}^{2}-2 \int_{\mathcal{F}_{h}} \llbracket v \rrbracket_{T} \cdot\left\{\nabla_{h} \times \boldsymbol{v}\right\} \mathrm{d} A+\sum_{F \in \mathcal{F}_{h}}\left(n_{f}+\eta_{F}\right)\left\|\mathcal{R}_{F}\left(\llbracket v \rrbracket_{T}\right)\right\|_{0}^{2} \\
& \quad \geq \beta^{2}\left(\left\|\nabla_{h} \times \boldsymbol{v}\right\|_{0}^{2}+\left\|\mathrm{h}^{-\frac{1}{2}} \llbracket v \rrbracket_{T}\right\|_{0, \mathcal{F}_{h}}^{2}\right) .
\end{aligned}
$$

The second term on the left hand side can be estimated with any positive $C_{K^{L}}$ and $C_{K^{R}}$ as,

$$
\begin{aligned}
& 2 \int_{\mathcal{F}_{h}} \llbracket v \rrbracket_{T} \cdot\left\{\nabla_{h} \times v \boldsymbol{d} A\right. \\
& =\sum_{F \in \mathcal{F}_{h}} \int_{F} \frac{2}{\sqrt{1-\beta^{2}}} h_{F}^{-\frac{1}{2}} C_{K^{L}}^{-1} \llbracket v \rrbracket_{T} \cdot C_{K^{L}} \frac{\sqrt{1-\beta^{2}}}{2} h_{F}^{\frac{1}{2}} \nabla_{h} \times\left.\boldsymbol{v}^{L}\right|_{F} \\
& \quad+\frac{2}{\sqrt{1-\beta^{2}}} h_{F}^{-\frac{1}{2}} C_{K^{R}}^{-1} \llbracket v \rrbracket_{T} \cdot C_{K^{R}} \frac{\sqrt{1-\beta^{2}}}{2} h_{F}^{\frac{1}{2}} \nabla_{h} \times\left. v^{R}\right|_{F} \mathrm{~d} A \\
& \leq \frac{1}{1-\beta^{2}} \sum_{F \in \mathcal{F}_{h}} h_{F}^{-1} C_{K^{L}}^{-2}\left\|\llbracket v \rrbracket_{T}\right\|_{0, F}^{2}+\frac{1-\beta^{2}}{4} \sum_{F \in \mathcal{F}_{h}} h_{F} C_{K^{L}}^{2}\left\|\nabla_{h} \times \boldsymbol{v}^{L}\right\|_{0, F}^{2} \\
& \quad+\frac{1}{1-\beta^{2}} \sum_{F \in \mathcal{F}_{h}} h_{F}^{-1} C_{K^{R}}^{-2}\left\|\llbracket v \rrbracket_{T}\right\|_{0, F}^{2}+\frac{1-\beta^{2}}{4} \sum_{F \in \mathcal{F}_{h}} h_{F} C_{K^{R}}^{2}\left\|\nabla_{h} \times \boldsymbol{v}^{R}\right\|_{0, F}^{2} .
\end{aligned}
$$


Applying (42) to the curl terms on the right-hand side of (46), we obtain

$$
\frac{1-\beta^{2}}{4} h_{F} C_{K^{L}}^{2}\left\|\nabla_{h} \times v^{L}\right\|_{0, F}^{2} \leq \frac{1-\beta^{2}}{4} h_{F} C_{K^{L}}^{2} \frac{(p+1)(p+3)}{3} \frac{S(F)}{V\left(K^{L}\right)}\left\|\nabla_{h} \times v^{L}\right\|_{0, K^{L}}^{2},
$$

and in the same way

$$
\frac{1-\beta^{2}}{4} h_{F} C_{K^{R}}^{2}\left\|\nabla_{h} \times v^{R}\right\|_{0, F}^{2} \leq \frac{1-\beta^{2}}{4} h_{F} C_{K^{R}}^{2} \frac{(p+1)(p+3)}{3} \frac{S(F)}{V\left(K^{R}\right)}\left\|\nabla_{h} \times v^{R}\right\|_{0, K^{R}}^{2} .
$$

For the jump terms, using (26), we obtain

$$
C_{K^{L}}^{-2} h_{F}^{-1}\left\|\llbracket v \rrbracket_{T}\right\|_{0, F}^{2} \leq C_{K^{L}}^{-2} h_{F}^{-1} \frac{3}{2} \frac{V\left(K^{L}\right)}{S(F)} \frac{F^{2}(p)}{p^{2}}\left\|\mathcal{R}_{F}\left(\llbracket v \rrbracket_{T}\right)\right\|_{0}^{2},
$$

and in the same way

$$
C_{K^{R}}^{-2} h_{F}^{-1}\left\|\llbracket v \rrbracket_{T}\right\|_{0, F}^{2} \leq C_{K^{R}}^{-2} h_{F}^{-1} \frac{3}{2} \frac{V\left(K^{R}\right)}{S(F)} \frac{F^{2}(p)}{p^{2}}\left\|\mathcal{R}_{F}\left(\llbracket v \rrbracket_{T}\right)\right\|_{0}^{2} .
$$

Choosing

$$
C_{K^{L}}=\sqrt{\frac{3 V\left(K^{L}\right)}{h_{F}(p+1)(p+3) S(F)}} \text { and } \quad C_{K^{R}}=\sqrt{\frac{3 V\left(K^{R}\right)}{h_{F}(p+1)(p+3) S(F)}}
$$

respectively, and summation of the inequalities in (47) and (48) (for all of the four faces of all tetrahedra) gives that

$$
\begin{aligned}
& \frac{1-\beta^{2}}{4} \sum_{F \in \mathcal{F}_{h}} h_{F} C_{K^{L}}^{2}\left\|\nabla_{h} \times \boldsymbol{v}^{L}\right\|_{0, F}^{2}+\frac{1-\beta^{2}}{4} \sum_{F \in \mathcal{F}_{h}} h_{F} C_{K^{R}}^{2}\left\|\nabla_{h} \times \boldsymbol{v}^{R}\right\|_{0, F}^{2} \\
& \quad \leq\left(1-\beta^{2}\right)\left\|\nabla_{h} \times \boldsymbol{v}\right\|_{0}^{2}
\end{aligned}
$$

and similarly, summation of (49) and (50) gives that

$$
\begin{aligned}
& \frac{1}{1-\beta^{2}} \sum_{F \in \mathcal{F}_{h}} C_{K^{L}}^{-2} h_{F}^{-1}\left\|\llbracket v \rrbracket_{T}\right\|_{0, F}^{2}+\frac{1}{1-\beta^{2}} \sum_{F \in \mathcal{F}_{h}} C_{K}^{-2} h_{F}^{-1}\left\|\llbracket v \rrbracket_{T}\right\|_{0, F}^{2} \\
& \leq \frac{1}{1-\beta^{2}} \frac{F^{2}(p)(p+1)(p+3)}{p^{2}}\left\|\mathcal{R}\left(\llbracket v \rrbracket_{T}\right)\right\|_{0}^{2} .
\end{aligned}
$$

Using estimates (51) and (52) in (46) we obtain that

$$
\begin{aligned}
& 2 \int_{\mathcal{F}_{h}} \llbracket \boldsymbol{v} \rrbracket_{T} \cdot\left\{\nabla_{h} \times \boldsymbol{v}\right\} \mathrm{d} A \\
& \quad \leq \frac{1}{1-\beta^{2}} \frac{F^{2}(p)(p+1)(p+3)}{p^{2}}\left\|\mathcal{R}\left(\llbracket v \rrbracket_{T}\right)\right\|_{0}^{2}+\left(1-\beta^{2}\right)\left\|\nabla_{h} \times v\right\|_{0}^{2} .
\end{aligned}
$$

Therefore, using also (41) we can estimate the left hand side of (45) as 


$$
\begin{aligned}
& \left\|\nabla_{h} \times \boldsymbol{v}\right\|_{0}^{2}-2 \int_{\mathcal{F}_{h}} \llbracket v \rrbracket_{T} \cdot\left\{\nabla_{h} \times \boldsymbol{v}\right\} \mathrm{d} A+\sum_{F \in \mathcal{F}_{h}}\left(n_{f}+\eta_{F}\right)\left\|\mathcal{R}_{F}\left(\llbracket v \rrbracket_{T}\right)\right\|_{0}^{2} \\
& \geq \beta^{2}\left\|\nabla_{h} \times \boldsymbol{v}\right\|_{0}^{2}+\sum_{F \in \mathcal{F}_{h}}\left(n_{f}+\eta_{F}-\frac{1}{1-\beta^{2}} \frac{F^{2}(p)(p+1)(p+3)}{p^{2}}\right)\left\|\mathcal{R}_{F}\left(\llbracket v \rrbracket_{T}\right)\right\|_{0}^{2} \\
& \geq \beta^{2}\left\|\nabla_{h} \times \boldsymbol{v}\right\|_{0}^{2} \\
& \quad+\sum_{F \in \mathcal{F}_{h}} h_{F}\left(n_{f}+\eta_{F}-\frac{1}{1-\beta^{2}} \frac{F^{2}(p)(p+1)(p+3)}{p^{2}}\right) \\
& \quad \times \frac{p^{2}}{F^{2}(p)} \frac{2}{3} M_{F} h_{F}^{-1}\left\|\llbracket v \rrbracket_{T}\right\|_{0}^{2} .
\end{aligned}
$$

Therefore, we have to choose $\eta_{F}$ such that

$$
h_{F} M_{F} \cdot\left(n_{f}+\eta_{F}-\frac{1}{1-\beta^{2}} \frac{F^{2}(p)(p+1)(p+3)}{p^{2}}\right) \frac{2}{3} \frac{p^{2}}{F^{2}(p)} \geq \beta^{2}
$$

and with this (45) is satisfied.

\section{Remarks}

1. Given that $n_{f}=4$ for tetrahedra we can make the condition for $\eta_{F}$ explicit,

$$
\eta_{F, 0}=\frac{F^{2}(p)}{p^{2}}\left(\frac{3 \beta^{2}}{2 h_{F} M_{F}}+\frac{(p+1)(p+3)}{1-\beta^{2}}\right)-4 .
$$

2. The coercivity constant $\beta$ is, however, still undefined. Using the a priori error analysis, which will be discussed in the next section, we can find an optimal value for $\eta_{F, 0}$.

3. A straightforward estimation gives that $\frac{F^{2}(p)(p+1)(p+3)}{p^{2}} \geq 1$, which together with (55) gives that

$$
n_{f}+\eta_{F} \geq 1 \quad \text { if } 0 \leq \beta^{2}<1 .
$$

Observe that for an arbitrary $K$ we have diam $K=h_{F} \geq m_{F}$, where $F$ is a face of $K$ and $m_{F}$ is the height corresponding to $F$. Hence,

$$
S(F) h_{F} \geq S(F) m_{F}=3 V(K)
$$

and therefore,

$$
\max _{F \in \mathcal{F}_{h}} h_{F} M_{F} \geq \max _{F \in \mathcal{F}_{h}} h_{F} \max \left\{\frac{S(F)}{V\left(K^{L}\right)}, \frac{S(F)}{V\left(K^{R}\right)}\right\} \geq 3 .
$$

Using the method in Lemma 3 we can also obtain a bound for the penalty parameter in the interior penalty (IP) method (18) such that the Gårding inequality is valid.

Lemma 4 There exist constants $\mathrm{a}_{F, 0}$, independent of the discretisation parameter $h=$ $\max _{K \in \mathcal{T}_{h}} \operatorname{diam} K$ and the wave number $k$, such that for all $\boldsymbol{v} \in \Sigma_{h}^{p}$ and all parameters $\mathrm{a}_{F} \geq \mathrm{a}_{F, 0}$ we have the following inequality

$$
\mathcal{B}_{h}^{i p}(\boldsymbol{v}, \boldsymbol{v}) \geq \beta^{2}\|\boldsymbol{v}\|_{\mathrm{DG}}^{2}-\left(k^{2}+\beta^{2}\right)\|\boldsymbol{v}\|_{0}^{2} .
$$


Proof According to the proof of Lemma 3 it is sufficient to prove that

$$
\begin{aligned}
& \left\|\nabla_{h} \times \boldsymbol{v}\right\|_{0}^{2}-2 \int_{\mathcal{F}_{h}} \llbracket v \rrbracket_{T} \cdot\left\{\nabla_{h} \times \boldsymbol{v}\right\} \mathrm{d} A+\sum_{F \in \mathcal{F}_{h}} \mathrm{a}_{F}\left\|\llbracket v \rrbracket_{T}\right\|_{0, F}^{2} \\
& \geq \beta^{2}\left(\left\|\nabla_{h} \times \boldsymbol{v}\right\|_{0}^{2}+\left\|\mathrm{h}^{-\frac{1}{2}} \llbracket \boldsymbol{v} \rrbracket_{T}\right\|_{0, \mathcal{F}_{h}}^{2}\right) .
\end{aligned}
$$

With the same choice of coefficients $C_{K^{L}}$ and $C_{K^{R}}$ as in Lemma 3 and using (51), we obtain the inequality

$$
\begin{aligned}
& 2 \int_{\mathcal{F}_{h}} \llbracket \boldsymbol{v} \rrbracket_{T} \cdot \llbracket \nabla_{h} \times \boldsymbol{v} \rrbracket \mathrm{d} A \\
& \leq \frac{1}{1-\beta^{2}} \sum_{F \in \mathcal{F}_{h}} C_{K^{L}}^{-2} h_{F}^{-1}\left\|\llbracket v \rrbracket_{T}\right\|_{0, F}^{2} \\
& \quad+\frac{1}{1-\beta^{2}} \sum_{F \in \mathcal{F}_{h}} C_{K^{R}}^{-2} h_{F}^{-1}\left\|\llbracket v \rrbracket_{T}\right\|_{0, F}^{2}+\left(1-\beta^{2}\right)\left\|\nabla_{h} \times \boldsymbol{v}\right\|_{0}^{2} \\
& \quad \leq \frac{1}{1-\beta^{2}} \frac{(p+1)(p+3)}{3}\left(\frac{S(F)}{V\left(K^{L}\right)}+\frac{S(F)}{V\left(K^{R}\right)}\right)\left\|\llbracket v \rrbracket_{T}\right\|_{\mathcal{F}_{h}, 0}^{2}+\left(1-\beta^{2}\right)\left\|\nabla_{h} \times v\right\|_{0}^{2}
\end{aligned}
$$

Substituting (46) into the right-hand side and using also (51), the left hand side of (60) is estimated as

$$
\begin{aligned}
& \left\|\nabla_{h} \times \boldsymbol{v}\right\|_{0}^{2}-2 \int_{\mathcal{F}_{h}} \llbracket v \rrbracket_{T} \cdot \llbracket \nabla_{h} \times \boldsymbol{v} \rrbracket \mathrm{d} A+\sum_{F \in \mathcal{F}_{h}} \mathrm{a}_{F}\left\|\llbracket v \rrbracket_{T}\right\|_{0}^{2} \\
& \geq \beta^{2}\left\|\nabla_{h} \times \boldsymbol{v}\right\|_{0}^{2} \\
& \quad+\sum_{F \in \mathcal{F}_{h}} h_{F}\left(\mathrm{a}_{F}-\frac{1}{1-\beta^{2}} \frac{(p+1)(p+3)}{3}\left(\frac{S(F)}{V\left(K^{L}\right)}+\frac{S(F)}{V\left(K^{R}\right)}\right)\right) h_{F}^{-1}\left\|\llbracket v \rrbracket_{T}\right\|_{\mathcal{F}_{h}, 0}^{2} .
\end{aligned}
$$

We have to choose then the parameter $\mathrm{a}_{F}$ on the face $F$ such that

$$
h_{F}\left(a_{F}-\frac{1}{1-\beta^{2}} \frac{1}{3}(p+1)(p+3)\left(\frac{S(F)}{V\left(K^{L}\right)}+\frac{S(F)}{V\left(K^{R}\right)}\right)\right) \geq \beta^{2},
$$

which gives the explicit bound

$$
\mathrm{a}_{F, 0} \geq \frac{\beta^{2}}{h_{F}}+\frac{1}{1-\beta^{2}} \frac{1}{3}(p+1)(p+3)\left(\frac{S(F)}{V\left(K^{L}\right)}+\frac{S(F)}{V\left(K^{R}\right)}\right) .
$$

This proves the lemma.

In the error analysis one has to consider (see [18]) the extended (cf. (23)) bilinear form

$$
\mathcal{B}^{b r}:\left(H_{0}(\operatorname{curl}, \Omega)+\Sigma_{h}^{p}\right) \times\left(H_{0}(\operatorname{curl}, \Omega)+\Sigma_{h}^{p}\right) \rightarrow \mathbb{R},
$$

which is given as

$$
\begin{aligned}
\mathcal{B}^{b r}(\boldsymbol{u}, \boldsymbol{v})= & \left(\nabla_{h} \times \boldsymbol{u}, \nabla_{h} \times \boldsymbol{v}\right)_{\Omega}-k^{2}(\boldsymbol{u}, \boldsymbol{v})_{\Omega}-\sum_{F \in \mathcal{F}_{h}}\left(\mathcal{R}_{F}\left(\llbracket \boldsymbol{u} \rrbracket_{T}\right), \nabla_{h} \times \boldsymbol{v}\right)_{\Omega} \\
& -\left(\mathcal{R}_{F}\left(\llbracket \boldsymbol{v} \rrbracket_{T}\right), \nabla_{h} \times \boldsymbol{u}\right)_{\Omega}+\sum_{F \in \mathcal{F}_{h}}\left(n_{f}+\eta_{F}\right)\left(\mathcal{R}_{F}\left(\llbracket \boldsymbol{u} \rrbracket_{T}\right), \mathcal{R}_{F}\left(\llbracket \boldsymbol{v} \rrbracket_{T}\right)\right)_{\Omega}
\end{aligned}
$$


and the linear form $\mathcal{J}_{h}: H_{0}(\operatorname{curl}, \Omega)+\Sigma_{h}^{p} \rightarrow \mathbb{R}$, defined as

$$
\mathcal{J}_{h}(\boldsymbol{v})=(\boldsymbol{J}, \boldsymbol{v})_{\Omega}
$$

when zero boundary conditions are considered. In following two lemmas we use the notation

$$
\mathcal{M}=\max _{F \in \mathcal{F}_{h}} \sqrt{h_{F} M_{F} \frac{(p+1)(p+3)}{6}} .
$$

Using (58) for $p \geq 1$ we have that $\mathcal{M} \geq 2$.

Using the inverse trace inequality (42) we also have that

$$
\begin{aligned}
\left\|\nabla_{h} \times \boldsymbol{u}^{L}\right\|_{0, F}^{2} & \leq \frac{(p+1)(p+3)}{3} \frac{S(F)}{V\left(K^{L}\right)}\left\|\nabla_{h} \times \boldsymbol{u}\right\|_{0, K^{L}}^{2} \\
& \leq h_{F}^{-1} \max _{F \in \mathcal{F}_{h}} M_{F} h_{F} \frac{(p+1)(p+3)}{3}\left\|\nabla_{h} \times \boldsymbol{u}\right\|_{0, K^{L}}^{2} \\
& \leq 2 h_{F}^{-1} \mathcal{M}^{2}\left\|\nabla_{h} \times \boldsymbol{u}\right\|_{0, K^{L}}^{2}
\end{aligned}
$$

and a similar estimate holds for the neighbouring element $K^{R}$.

Lemma 5 The bilinear form $\mathcal{B}^{b r}$ is continuous on $\left(H_{0}(\operatorname{curl}, \Omega)+\Sigma_{h}^{p}\right) \times\left(H_{0}(\operatorname{curl}, \Omega)+\Sigma_{h}^{p}\right)$ with respect to the $D G$ norm, i.e. the following inequality holds for all $\boldsymbol{u}=\boldsymbol{u}_{0}+\boldsymbol{u}_{h}$ and $\boldsymbol{v}=\boldsymbol{v}_{0}+\boldsymbol{v}_{h}$ with $\boldsymbol{u}_{0}, \boldsymbol{v}_{0} \in H_{0}(\operatorname{curl}, \Omega)$ and $\boldsymbol{u}_{h}, \boldsymbol{v}_{h} \in \Sigma_{h}^{p}$ :

$$
\mathcal{B}^{b r}(\boldsymbol{u}, \boldsymbol{v}) \leq C\|\boldsymbol{u}\|_{\mathrm{DG}}\|\boldsymbol{v}\|_{\mathrm{DG}},
$$

where

$$
C=\max _{F \in \mathcal{F}_{h}}\left\{k^{2}, \frac{5}{4} \mathcal{M}^{2}\left(n_{f}+\eta_{F}\right)\right\} .
$$

Proof Using the triangle inequality, Lemma 2, the result of the eigenvalue problem discussed in the Appendix, the estimate $\mathcal{M} \geq 2$ and (57) we obtain that

$$
\begin{aligned}
\mathcal{B}^{b r}(\boldsymbol{u}, \boldsymbol{v}) \leq & \left|\left(\nabla_{h} \times \boldsymbol{u}, \nabla_{h} \times \boldsymbol{v}\right)_{\Omega}\right|+k^{2}\left|(\boldsymbol{u}, \boldsymbol{v})_{\Omega}\right|+\sum_{F \in \mathcal{F}_{h}}\left|\left(\mathcal{R}_{F}\left(\llbracket \boldsymbol{u} \rrbracket_{T}\right), \nabla_{h} \times \boldsymbol{v}\right)_{\Omega}\right| \\
& +\left|\left(\mathcal{R}_{F}\left(\llbracket \boldsymbol{v} \rrbracket_{T}\right), \nabla_{h} \times \boldsymbol{u}\right)_{\Omega}\right|+\left|\sum_{F \in \mathcal{F}_{h}}\left(n_{f}+\eta_{F}\right)\left(\mathcal{R}_{F}\left(\llbracket \boldsymbol{u} \rrbracket_{T}\right), \mathcal{R}_{F}\left(\llbracket \boldsymbol{v} \rrbracket_{T}\right)\right)_{\Omega}\right| \\
\leq & \left\|\nabla_{h} \times \boldsymbol{u}\right\|_{0}\left\|\nabla_{h} \times \boldsymbol{v}\right\|_{0}+k^{2}\|\boldsymbol{u}\|_{0}\|\boldsymbol{v}\|_{0} \\
& +\sum_{F \in \mathcal{F}_{h}}\left\|\mathcal{R}_{F}\left(\llbracket \boldsymbol{u} \rrbracket_{T}\right)\right\|_{0}\left\|\nabla_{h} \times \boldsymbol{v}\right\|_{0} \\
& +\sum_{F \in \mathcal{F}_{h}}\left\|\mathcal{R}_{F}\left(\llbracket v \rrbracket_{T}\right)\right\|_{0}\left\|\nabla_{h} \times \boldsymbol{u}\right\|_{0} \\
& +\sum_{F \in \mathcal{F}_{h}}\left(n_{f}+\eta_{F}\right)\left\|\mathcal{R}_{F}\left(\llbracket \boldsymbol{u} \rrbracket_{T}\right)\right\|_{0}\left\|\mathcal{R}_{F}\left(\llbracket \boldsymbol{v} \rrbracket_{T}\right)\right\|_{0}
\end{aligned}
$$




$$
\begin{aligned}
\leq & \left\|\nabla_{h} \times \boldsymbol{u}\right\|_{0}\left\|\nabla_{h} \times \boldsymbol{v}\right\|_{0}+k^{2}\|\boldsymbol{u}\|_{0}\|\boldsymbol{v}\|_{0} \\
& +\mathcal{M}\left\|\mathrm{h}_{F}^{-\frac{1}{2}} \llbracket \boldsymbol{u} \rrbracket_{T}\right\|_{0, \mathcal{F}_{h}}\left\|\nabla_{h} \times \boldsymbol{v}\right\|_{0}+\mathcal{M}\left\|\mathrm{h}_{F}^{-\frac{1}{2}} \llbracket \boldsymbol{v} \rrbracket_{T}\right\|_{0, \mathcal{F}_{h}}\left\|\nabla_{h} \times \boldsymbol{u}\right\|_{0} \\
& +\mathcal{M}^{2}\left\|\mathrm{~h}_{F}^{-\frac{1}{2}} \llbracket \boldsymbol{u} \rrbracket_{T}\right\|_{0, \mathcal{F}_{h}}\left\|\mathrm{~h}_{F}^{-\frac{1}{2}} \llbracket \boldsymbol{v} \rrbracket_{T}\right\|_{0, \mathcal{F}_{h}} \max _{F}\left(n_{f}+\eta_{F}\right) \\
\leq & \max _{F \in \mathcal{F}_{h}}\left\{k^{2}, 1+\mathcal{M}^{2}\left(n_{f}+\eta_{F}\right)\right\}\|\boldsymbol{u}\|_{\mathrm{DG}}\|\boldsymbol{v}\|_{\mathrm{DG}} \\
\leq & \max _{F \in \mathcal{F}_{h}}\left\{k^{2}, \frac{5}{4} \mathcal{M}^{2}\left(n_{f}+\eta_{F}\right)\right\}\|\boldsymbol{u}\|_{\mathrm{DG}}\|\boldsymbol{v}\|_{\mathrm{DG}},
\end{aligned}
$$

which was stated in the lemma.

The fourth inequality in the previous lemma is obtained by solving a simple eigenvalue problem, relegated to the Appendix for the sake of readability.

A similar result can be proved for the IP method. In the analysis of the IP method one should again extend the discretisation operator $\mathcal{B}^{i p}$ to $\left(H_{0}(\operatorname{curl}, \Omega)+\Sigma_{h}^{p}\right) \times\left(H_{0}(\operatorname{curl}, \Omega)+\right.$ $\left.\Sigma_{h}^{p}\right) \rightarrow \mathbb{R}$, see [18], and with this the following estimate is valid.

Lemma 6 The bilinear form $\mathcal{B}^{i p}$ is continuous on $\left(H_{0}(\operatorname{curl}, \Omega)+\Sigma_{h}^{p}\right) \times\left(H_{0}(\operatorname{curl}, \Omega)+\Sigma_{h}^{p}\right)$ with respect to the $D G$ norm, i.e. the following inequality holds for all $\boldsymbol{u}=\boldsymbol{u}_{0}+\boldsymbol{u}_{h}$ and $\boldsymbol{v}=\boldsymbol{v}_{0}+\boldsymbol{v}_{h}$ with $\boldsymbol{u}_{0}, \boldsymbol{v}_{0} \in H_{0}(\operatorname{curl}, \Omega)$ and $\boldsymbol{u}_{h}, \boldsymbol{v}_{h} \in \Sigma_{h}^{p}$ :

$$
\mathcal{B}^{i p}(\boldsymbol{u}, \boldsymbol{v}) \leq C\|\boldsymbol{u}\|_{\mathrm{DG}}\|\boldsymbol{v}\|_{\mathrm{DG}},
$$

where

$$
C=\max _{F \in \mathcal{F}_{h}}\left\{k^{2}, h_{F} \mathrm{a}_{F}+\frac{3}{2} \mathcal{M}\right\} .
$$

Proof Using the triangle inequality and (62), we obtain that

$$
\begin{aligned}
\mathcal{B}^{i p}(\boldsymbol{u}, \boldsymbol{v}) \leq & \left\|\nabla_{h} \times \boldsymbol{u}\right\|_{0}\left\|\nabla_{h} \times \boldsymbol{v}\right\|_{0}+k^{2}\|\boldsymbol{u}\|_{0}\|\boldsymbol{v}\|_{0} \\
& +\sum_{F \in \mathcal{F}_{h}}\left\|\llbracket \boldsymbol{u} \rrbracket_{T}\right\|_{0, F}\left\|\frac{1}{2}\left(\nabla_{h} \times \boldsymbol{v}^{L}+\nabla_{h} \times \boldsymbol{v}^{R}\right)\right\|_{0, F} \\
& +\sum_{F \in \mathcal{F}_{h}}\left\|\llbracket \boldsymbol{v} \rrbracket_{T}\right\|_{0, F}\left\|\frac{1}{2}\left(\nabla_{h} \times \boldsymbol{u}^{L}+\nabla_{h} \times \boldsymbol{u}^{R}\right)\right\|_{0, F} \\
& +\sum_{F \in \mathcal{F}_{h}} \mathrm{a}_{F}\left\|\llbracket \boldsymbol{u} \rrbracket_{T}\right\|_{0, F}\left\|\llbracket \boldsymbol{v} \rrbracket_{T}\right\|_{0, F} \\
\leq & \left\|\nabla_{h} \times \boldsymbol{u}\right\|_{0}\left\|\nabla_{h} \times \boldsymbol{v}\right\|_{0}+k^{2}\|\boldsymbol{u}\|_{0}\|\boldsymbol{v}\|_{0} \\
& +\left\|\mathrm{h}^{-\frac{1}{2}} \llbracket \boldsymbol{u} \rrbracket_{T}\right\|_{0, \mathcal{F}_{h}}\left(\sum_{F \in \mathcal{F}_{h}} h_{F}\left\|\frac{1}{2}\left(\nabla_{h} \times \boldsymbol{u}^{L}+\nabla_{h} \times \boldsymbol{u}^{R}\right)\right\|_{0, F}^{2}\right)^{\frac{1}{2}} \\
& +\left\|\mathrm{h}^{-\frac{1}{2}} \llbracket \boldsymbol{v} \rrbracket_{T}\right\|_{0, \mathcal{F}_{h}}\left(\sum_{F \in \mathcal{F}_{h}} h_{F}\left\|\frac{1}{2}\left(\nabla_{h} \times \boldsymbol{v}^{L}+\nabla_{h} \times \boldsymbol{v}^{R}\right)\right\|_{0, F}^{2}\right)^{\frac{1}{2}} \\
& +\sum_{F \in \mathcal{F}_{h}} h_{F} \mathrm{a}_{F} \cdot h_{F}^{-\frac{1}{2}}\left\|\llbracket \boldsymbol{u} \rrbracket_{T}\right\|_{0, F} \cdot h_{F}^{-\frac{1}{2}}\left\|\llbracket \boldsymbol{v} \rrbracket_{T}\right\|_{0, F}
\end{aligned}
$$




$$
\begin{aligned}
& \leq\left\|\nabla_{h} \times \boldsymbol{u}\right\|_{0}\left\|\nabla_{h} \times \boldsymbol{v}\right\|_{0}+k^{2}\|\boldsymbol{u}\|_{0}\|\boldsymbol{v}\|_{0} \\
& +\left\|\mathrm{h}^{-\frac{1}{2}} \llbracket \boldsymbol{u} \rrbracket_{T}\right\|_{0, \mathcal{F}_{h}}\left(\sum_{F \in \mathcal{F}_{h}} \frac{h_{F}}{2}\left\|\nabla_{h} \times \boldsymbol{u}^{L}\right\|_{0, F}^{2}+\frac{h_{F}}{2}\left\|\nabla_{h} \times \boldsymbol{u}^{R}\right\|_{0, F}^{2}\right)^{\frac{1}{2}} \\
& +\left\|\mathrm{h}^{-\frac{1}{2}} \llbracket \boldsymbol{v} \rrbracket_{T}\right\|_{0, \mathcal{F}_{h}}\left(\sum_{F \in \mathcal{F}_{h}} \frac{h_{F}}{2}\left\|\nabla_{h} \times \boldsymbol{v}^{L}\right\|_{0, F}^{2}+\frac{h_{F}}{2}\left\|\nabla_{h} \times \boldsymbol{v}^{R}\right\|_{0, F}^{2}\right)^{\frac{1}{2}} \\
& +\sum_{F \in \mathcal{F}_{h}} h_{F} \mathrm{a}_{F} \cdot h_{F}^{-\frac{1}{2}}\left\|\llbracket \boldsymbol{u} \rrbracket_{T}\right\|_{0, F} \cdot h_{F}^{-\frac{1}{2}}\left\|\llbracket v \rrbracket_{T}\right\|_{0, F} \\
& \leq\left\|\nabla_{h} \times \boldsymbol{u}\right\|_{0}\left\|\nabla_{h} \times \boldsymbol{v}\right\|_{0}+k^{2}\|\boldsymbol{u}\|_{0}\|\boldsymbol{v}\|_{0} \\
& +\left\|\mathrm{h}^{-\frac{1}{2}} \llbracket \boldsymbol{u} \rrbracket_{T}\right\|_{0, \mathcal{F}_{h}}\left(\sum_{F \in \mathcal{F}_{h}} \mathcal{M}^{2}\left\|\nabla_{h} \times \boldsymbol{u}\right\|_{0, K^{L}}^{2}+\mathcal{M}^{2}\left\|\nabla_{h} \times \boldsymbol{u}\right\|_{0, K^{R}}^{2}\right)^{\frac{1}{2}} \\
& +\left\|\mathrm{h}^{-\frac{1}{2}} \llbracket \boldsymbol{v} \rrbracket_{T}\right\|_{0, \mathcal{F}_{h}}\left(\sum_{F \in \mathcal{F}_{h}} \mathcal{M}^{2}\left\|\nabla_{h} \times \boldsymbol{v}\right\|_{0, K^{L}}^{2}+\mathcal{M}^{2}\left\|\nabla_{h} \times \boldsymbol{v}\right\|_{0, K^{R}}^{2}\right)^{\frac{1}{2}} \\
& +\max _{F \in \mathcal{F}_{h}} h_{F} \mathrm{a}_{F} \sum_{F \in \mathcal{F}_{h}} h_{F}^{-\frac{1}{2}}\left\|\llbracket u \rrbracket_{T}\right\|_{0, F} \cdot h_{F}^{-\frac{1}{2}}\left\|\llbracket v \rrbracket_{T}\right\|_{0, F} \\
& \leq\left\|\nabla_{h} \times \boldsymbol{u}\right\|_{0}\left\|\nabla_{h} \times \boldsymbol{v}\right\|_{0}+k^{2}\|\boldsymbol{u}\|_{0}\|\boldsymbol{v}\|_{0}+2 \mathcal{M}\left\|\mathrm{h}^{-\frac{1}{2}} \llbracket \boldsymbol{u} \rrbracket_{T}\right\|_{0, \mathcal{F}_{h}}\left\|\nabla_{h} \times \boldsymbol{u}\right\|_{0} \\
& +2 \mathcal{M}\left\|\mathrm{h}^{-\frac{1}{2}} \llbracket \boldsymbol{v} \rrbracket_{T}\right\|_{0, \mathcal{F}_{h}}\left\|\nabla_{h} \times \boldsymbol{v}\right\|_{0} \\
& +\max _{F \in \mathcal{F}_{h}} h_{F} \mathrm{a}_{F}\left\|\mathrm{~h}^{-\frac{1}{2}} \llbracket \boldsymbol{u} \rrbracket_{T}\right\|_{0, \mathcal{F}_{h}}\left\|\mathrm{~h}^{-\frac{1}{2}} \llbracket v \rrbracket_{T}\right\|_{0, \mathcal{F}_{h}} \\
& \leq \max _{F \in \mathcal{F}_{h}}\left\{k^{2}, h_{F} \mathrm{a}_{F}+\frac{3}{2} \mathcal{M}\right\}\|\boldsymbol{u}\|_{\mathrm{DG}}\|\boldsymbol{v}\|_{\mathrm{DG}}
\end{aligned}
$$

as stated in the lemma.

The penultimate inequality is, again, the consequence of the a simple eigenvalue problem - see the Appendix-and the estimate $\max _{F \in \mathcal{F}} h_{F} \mathrm{a}_{F} \geq 1$, which can be proved using (61) with $\mathcal{M} \geq 2$.

Use now the Gårding inequality and the continuity of $\mathcal{B}^{b r}(\boldsymbol{u}, \boldsymbol{v})$ to obtain the following expression for the error,

$$
\begin{aligned}
\beta^{2}\left\|\boldsymbol{E}-\boldsymbol{E}_{h}\right\|_{\mathrm{DG}}^{2} \leq & \mathcal{B}_{h}^{b r}\left(\boldsymbol{E}-\boldsymbol{E}_{h}, \boldsymbol{E}-\boldsymbol{E}_{h}\right) \\
& +\left(k^{2}+\beta^{2}\right)\left\|\boldsymbol{E}-\boldsymbol{E}_{h}\right\|_{0, \Omega}^{2} \\
= & \mathcal{B}_{h}^{b r}\left(\boldsymbol{E}-\boldsymbol{E}_{h}, \boldsymbol{E}-\boldsymbol{v}\right) \\
& +\left(k^{2}+\beta^{2}\right)\left\|\boldsymbol{E}-\boldsymbol{E}_{h}^{2}\right\|_{0, \Omega}^{2} \\
\leq & \max _{F \in \mathcal{F}_{h}}\left\{k^{2}, \frac{5}{4} \mathcal{M}^{2}\left(n_{f}+\eta_{F}\right)\right\} \cdot\left\|\boldsymbol{E}-\boldsymbol{E}_{h}\right\|_{\mathrm{DG}}\|\boldsymbol{E}-\boldsymbol{v}\|_{\mathrm{DG}} \\
& +\left(k^{2}+\beta^{2}\right)\left\|\boldsymbol{E}-\boldsymbol{E}_{h}\right\|_{0, \Omega}^{2},
\end{aligned}
$$


where in the second line the orthogonality relation with $v \in \Sigma_{h}^{p}$ was used. From this we can arrive at the estimate

$$
\begin{aligned}
\left\|\boldsymbol{E}-\boldsymbol{E}_{h}\right\|_{\mathrm{DG}}^{2} \leq & \frac{1}{\beta^{2}} \max _{F \in \mathcal{F}_{h}}\left\{k^{2}, \frac{5}{4} \mathcal{M}^{2}\left(n_{f}+\eta_{F}\right)\right\} \inf _{\boldsymbol{v} \in \Sigma_{h}^{p}}\|\boldsymbol{E}-\boldsymbol{v}\|_{\mathrm{DG}}^{2} \\
& +\frac{k^{2}+\beta^{2}}{\beta^{2}}\left\|\boldsymbol{E}-\boldsymbol{E}_{h}\right\|_{0, \Omega}^{2} .
\end{aligned}
$$

Note that the coefficient

$$
M_{F} h_{F}=\max \left\{\frac{S(F)}{V\left(K^{L}\right)}, \frac{S(F)}{V\left(K^{R}\right)}\right\} h_{F}=\mathcal{O}(1),
$$

so the error depends on $k^{2}$, the polynomial order $p$ and the interpolation error, which in turn depends on $h$ and $p$. In addition, the coercivity constant $\beta$ plays an important part too and its value is related to the penalty parameter.

\subsection{Optimal Value for the Penalty Parameters}

The penalty parameter $\eta_{F}$ in the Brezzi DG formulation (25) and the coercivity constant $\beta$ in the Gårding inequality are related by (55) through

$$
\eta_{F} \geq \frac{3 F^{2}(p)}{2 p^{2} h_{F} M_{F}} \beta^{2}+\frac{1}{1-\beta^{2}} \frac{(p+1)(p+3)}{p^{2}} F^{2}(p)-n_{f},
$$

while according to (66) optimal accuracy requires a minimal coefficient

$$
\frac{1}{\beta^{2}} \max _{F \in \mathcal{F}_{h}}\left\{k^{2}, \frac{5}{4} \mathcal{M}^{2}\left(n_{f}+\eta_{F}\right)\right\} .
$$

Take now the minimum value for $\eta_{F}$ in (67) and use this in (68). For an optimal stabilisation, hence with a minimal effect on accuracy and efficiency, we need to minimise the second term in (68), i.e. the following quantity:

$$
\begin{aligned}
& \left(n_{f}+\eta_{F}\right) \frac{(p+1)(p+3)}{6 \beta^{2}} M_{F} h_{F} \\
& \quad=\left(\frac{3 F^{2}(p)}{2 p^{2} h_{F} M_{F}} \beta^{2}+\frac{1}{1-\beta^{2}} \frac{(p+1)(p+3)}{p^{2}} F^{2}(p)\right) \frac{(p+1)(p+3)}{6 \beta^{2}} M_{F} h_{F} .
\end{aligned}
$$

For this we can leave all constants and find $\beta$ that minimises the following

$$
\frac{1}{\beta^{2}\left(1-\beta^{2}\right)} \frac{(p+1)(p+3) F^{2}(p)}{p^{2}} .
$$

An elementary calculation gives that $\beta^{2}=\frac{1}{2}$ such that using (56) we obtain the optimal value of $\eta_{F}$ in $\mathcal{B}_{h}^{b r}$,

$$
\eta_{F, 0}=\frac{F^{2}(p)}{p^{2}}\left(\frac{3}{4 h_{F} M_{F}}+2(p+1)(p+3)\right)-4 .
$$


Analogously to the analysis for $\eta_{F}$ we can find an optimal value of $a_{F}$ using the relations (cf. (61))

$$
\mathrm{a}_{F} \geq \frac{\beta^{2}}{h_{F}}+\frac{1}{1-\beta^{2}} \frac{1}{3}(p+1)(p+3)\left(\frac{S(F)}{V\left(K^{L}\right)}+\frac{S(F)}{V\left(K^{R}\right)}\right)
$$

and minimise $\frac{h_{F} a_{F}}{\beta^{2}}$ in (64) with an appropriate $\beta$. Using (70) we obtain

$$
\frac{1}{2 h_{F}}+\frac{1}{\beta^{2}\left(1-\beta^{2}\right)} \frac{1}{3}(p+1)(p+3)\left(\frac{S(F)}{V\left(K^{L}\right)}+\frac{S(F)}{V\left(K^{R}\right)}\right)
$$

and again, we have a minimal value at $\beta^{2}=\frac{1}{2}$. The optimal value of $\mathrm{a}_{F}$ is thus

$$
\mathrm{a}_{F, 0}=\frac{1}{2 h_{F}}+\frac{2}{3}(p+1)(p+3)\left(\frac{S(F)}{V\left(K^{L}\right)}+\frac{S(F)}{V\left(K^{R}\right)}\right) .
$$

Note an interesting difference between the approximations using $\mathcal{B}^{i p}$ and $\mathcal{B}^{b r}$ is that $\mathrm{a}_{F}$ in the IP-DG method needs to be increased quadratically with the polynomial order, whereas in the DG method of the Brezzi type formulation

$$
\lim _{p \rightarrow \infty} \eta_{F}=8 \ln 2-4
$$

\subsection{Convergence of the Brezzi Type DG Method}

Using Lemmas 3 and 5 one can see that with obvious modifications the analysis in [18] can be carried out for the Brezzi type bilinear form and accordingly, we obtain the following.

Theorem 1 Assume that $\eta_{F}$ satisfies the condition in Lemma 3 and for some parameter $s>\frac{1}{2}$ the exact solution of (1) satisfies

$$
\boldsymbol{E} \in H^{s}(\Omega) \text { and } \nabla \times \boldsymbol{E} \in H^{s}(\Omega) .
$$

Then using a full polynomial finite element space of order $p$ with a mesh size $h$ sufficiently small, we have the following error bound

$$
\left\|\boldsymbol{E}-\boldsymbol{E}_{h}\right\|_{D G} \leq \beta^{-2} k^{2} C h^{\min \{p, s\}}\left(\|\boldsymbol{E}\|_{s}+\|\nabla \times \boldsymbol{E}\|_{s}\right),
$$

where the constant $C$ does not depend on $h$ and $k$.

\section{Remarks}

1. The $k$ and $\beta$ dependence of the constants can be obtained in the same way as in Proposition 5.1 in [18].

2. The constant $C$ in (72) depends on the coefficients in interpolation estimates, which can again depend on the geometry of the mesh and the polynomial order of the finite elements.

The results in [18] have been extended in [9], where a general framework is laid down to investigate the asymptotic spectral correctness of any DG discretisation of (2). Also, if a DG discretisation of (2) is spectrally correct (i.e. free of spurious modes), then the existence and uniqueness of the solution for the indefinite problem (1) is guaranteed. In order prove 
asymptotic spectral correctness, one only needs to check a set of conditions. These were proved for the symmetric IP-DG method in [9] on tetrahedral meshes and the results trivially extend to some other symmetric DG discretisations, including the Brezzi type considered in this article.

\section{Numerical Experiments}

The numerical examples in this section serve two purposes. First, they intend to show how sharp the parameter estimates are in the previous section. We will see how the $L^{2}$-error and the number of iterations (i.e. computational work) changes as a function of the penalty parameter for both the IP-DG method and the method of Brezzi et al. [8]. Second, we provide asymptotic convergence tests for both methods. Although we have little to add to the theoretical asymptotic results in $[9,18]$, our three-dimensional computations complement those results as they have so far been only verified on two-dimensional meshes [10, 11].

As a test example, we consider the Maxwell equations (1) with $k^{2}=1$ in the domain $\Omega=(0,1)^{3}$ and assume the boundary to be a perfect electric conductor (PEC), i.e. $\boldsymbol{g}=\mathbf{0}$ in (1). The source term is given as

$$
\boldsymbol{J}(x, y, z)=\left(2 \pi^{2}-1\right)\left(\begin{array}{l}
\sin (\pi y) \sin (\pi z) \\
\sin (\pi z) \sin (\pi x) \\
\sin (\pi x) \sin (\pi y)
\end{array}\right),
$$

so we have the exact solution

$$
\boldsymbol{E}(x, y, z)=\left(\begin{array}{l}
\sin (\pi y) \sin (\pi z) \\
\sin (\pi z) \sin (\pi x) \\
\sin (\pi x) \sin (\pi y)
\end{array}\right) .
$$

For all computations, a hierarchic construction of $H$ (curl)-conforming vector-valued basis functions is used $[1,27]$. The first six of the basis functions constitute the first-order firstfamily of Nédélec elements [22]. The first twelve of the basis functions used here are not the same as those that form the first-order second-family of Nédélec elements, defined in [23], but they span exactly the same space and have the same approximation properties as those.

All numerical computations have been carried out in the framework of $h p$ GEM [24], a software environment for DG discretisations suitable for a variety of physical problems. To solve the linear system that results from the DG discretisations, we use PETSc [3] and opt for MINRES as a suitable linear solver with incomplete Cholesky factorisation (ICC) ${ }^{1}$ as preconditioners.

\subsection{Sharpness of the Parameter Estimates}

In this example, we demonstrate the sharpness of the estimates (69) and (71). A range of different values of $\eta_{F}$ and $\mathrm{a}_{F}$ are used on two different meshes. One is a structured mesh of 320 tetrahedra and the other is an unstructured mesh of 432 tetrahedra. A tolerance of

\footnotetext{
${ }^{1}$ We note that ICC is not, in general, guaranteed to work for the discretisations considered here since the linear system is indefinite and Cholesky factorisation requires a positive definite matrix. However, it is successful in the following examples precisely because the factorisation is now incomplete.
} 
tol $=10^{-8}$ is used in MINRES but the linear solver is stopped after $10^{5}$ iterations even if that tolerance is not achieved.

For the DG method using the Brezzi formulation, we show the results on the structured mesh in Fig. 1 and on the unstructured mesh in Fig. 2. For the IP-DG method, Fig. 3 depicts the results on the structured mesh and Fig. 4 for the unstructured one. The critical parameter value is clearly visible in the plots for both methods: this is the point where the error as well as the iteration count drop dramatically. From here the error increases slightly as it converges to the error of the $H$ (curl)-conforming discretisation-where the tangential continuity is enforced strongly through the basis function rather than weakly through the penalty termof the same order. This convergence behaviour is a direct consequence of the theoretical and numerical study on the Maxwell eigenvalue problem in [29].

In contrast, the number of iterations increases indefinitely as the penalty parameters grow, resulting in excess computational cost. The increase is markedly steeper on the unstructured mesh than on the structured one. In each plot, the circles indicate the theoretical estimates (69) and (71), shown to be the optimal choice in the previous section. The theoretical estimates provide a clearly stable solution with computational cost no more than two times higher than the numerically established minimum. The estimate for the penalty parameter $\mathrm{a}_{F}$ of the IP-DG method is somewhat sharper than for the penalty parameter $\eta_{F}$ of the DG method with the Brezzi formulation. For both DG methods, the estimates for the higherorder polynomials, $p=3$ and, especially, $p=4$, are noticeably sharper. It is noteworthy that the estimate for $\mathrm{a}_{F}$ of the IP-DG method grows as we increase the polynomial order whereas for the DG method with the Brezzi formulation it is approximately constant. These properties are also reflected in the numerically established stability criterion.

\subsection{Asymptotic Convergence}

The theoretical framework for determining the asymptotic convergence rates of DG discretisations of the Maxwell equations is fairly complete in [9], albeit for conformal meshes. However, those theoretical results have so far been accompanied by two-dimensional computations only $[10,11,18]$. We now provide numerical three-dimensional convergence results for both DG methods discussed in this work.

The computations are performed on two different sequences of meshes. The first are highly structured meshes and constructed as follows. The domain $\Omega=(0,1)^{3}$ is divided into $n \times n \times n$ number of congruent subcubes, with integer $n=2^{m}$ and nonnegative integer $m$. We then divide each of these subcubes into five tetrahedra, four of which are congruent and have volume one-sixth of the original cube. The fifth has volume one-third of the original cube. Although the mesh is not uniform, this has proved to be a simple and convenient way of measuring convergence, as each time we refine the mesh, the maximum of the face diameter $h_{F}$ will be exactly half of that of the previous mesh. The convergence results on structured meshes are shown in Table 1 for the IP-DG method and in Table 3 for the DG method using the Brezzi formulation.

We have also run the same example on a sequence of unstructured meshes. The meshes were generated by CentaurSoft (http://www.centaursoft.com), a package suitable for generating a variety of hybrid meshes with complex geometries. In this sequence of meshes, we begin with a coarse mesh of 54 tetrahedra. Then we divide each tetrahedron into eight smaller tetrahedra to get the next (finer) mesh. The convergence results on unstructured meshes are depicted in Table 2 for the IP-DG discretisation and in Table 4 for the DG method using the Brezzi formulation. 

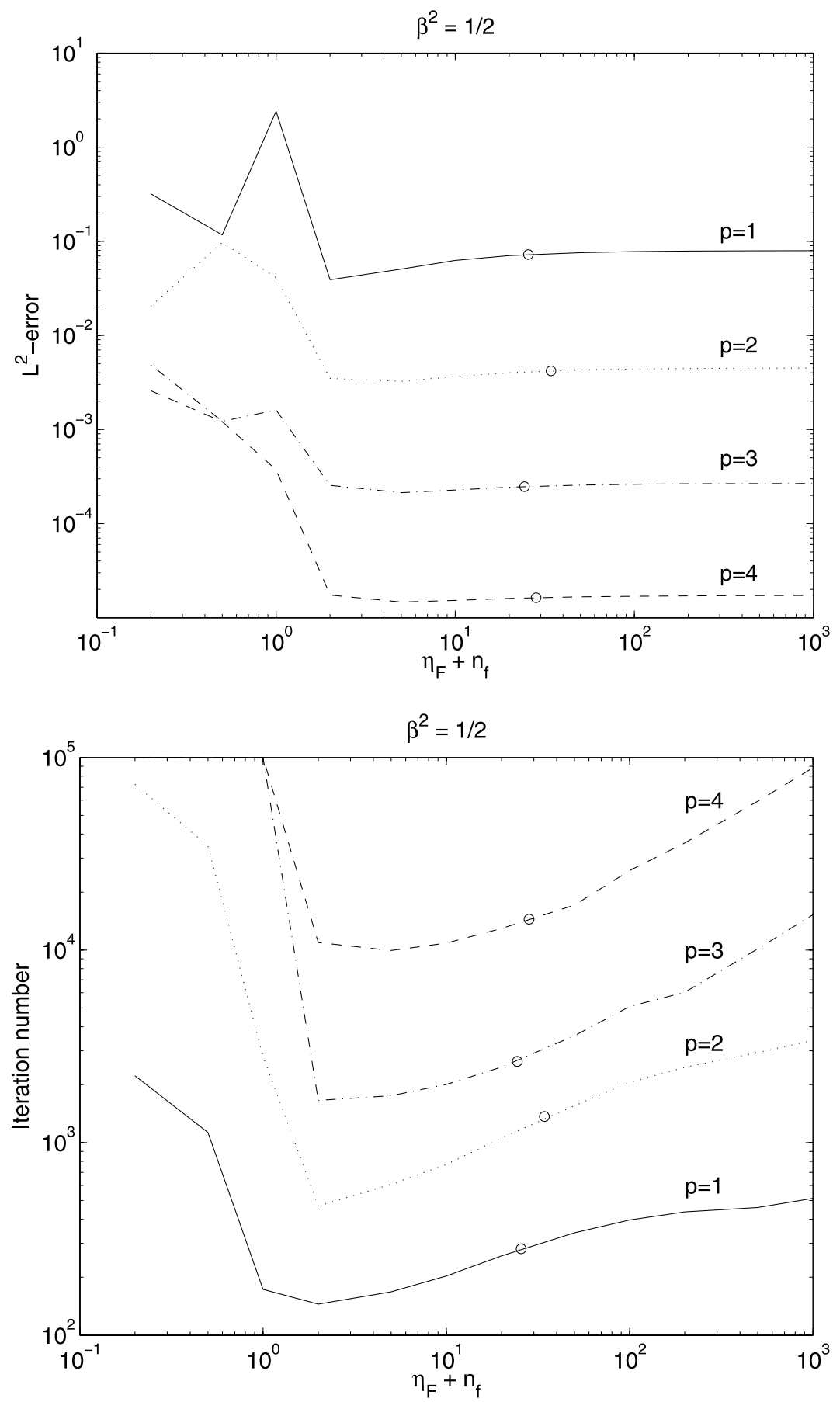

Fig. $1 L^{2}$-error (top) and the number of MINRES iterations (bottom) as a function of the penalty parameter $\eta_{F}+n_{f}$ in the DG formulation of Brezzi. A structured mesh of 320 tetrahedra and coercivity constant $\beta^{2}=\frac{1}{2}$ are used. The circles indicate the theoretical estimate (69) 

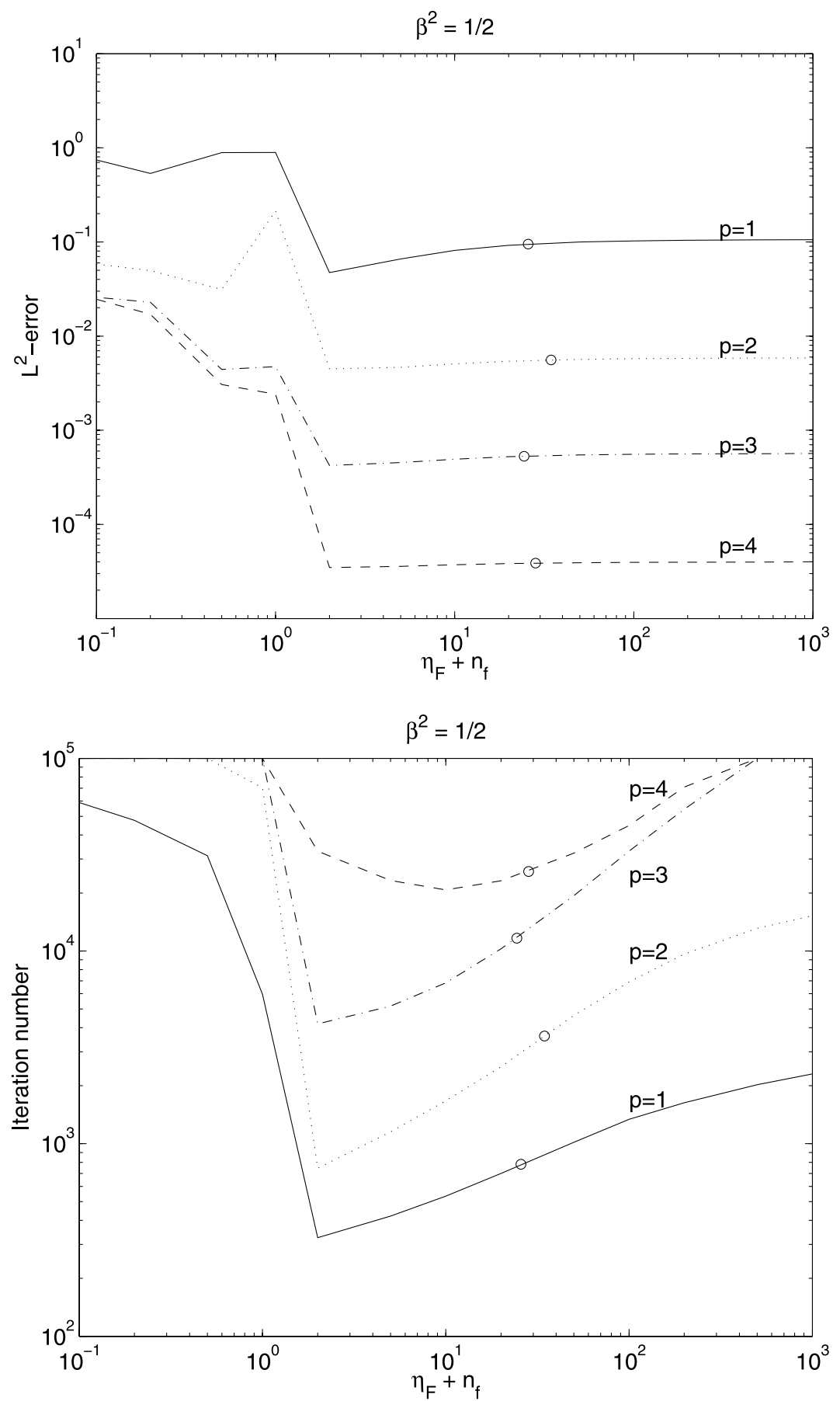

Fig. $2 L^{2}$-error (top) and the number of MINRES iterations (bottom) as a function of the penalty parameter $\eta_{F}+n_{f}$ in the DG formulation of Brezzi. An unstructured mesh of 432 tetrahedra and coercivity constant $\beta^{2}=\frac{1}{2}$ are used. The circles indicate the theoretical estimate (69) 

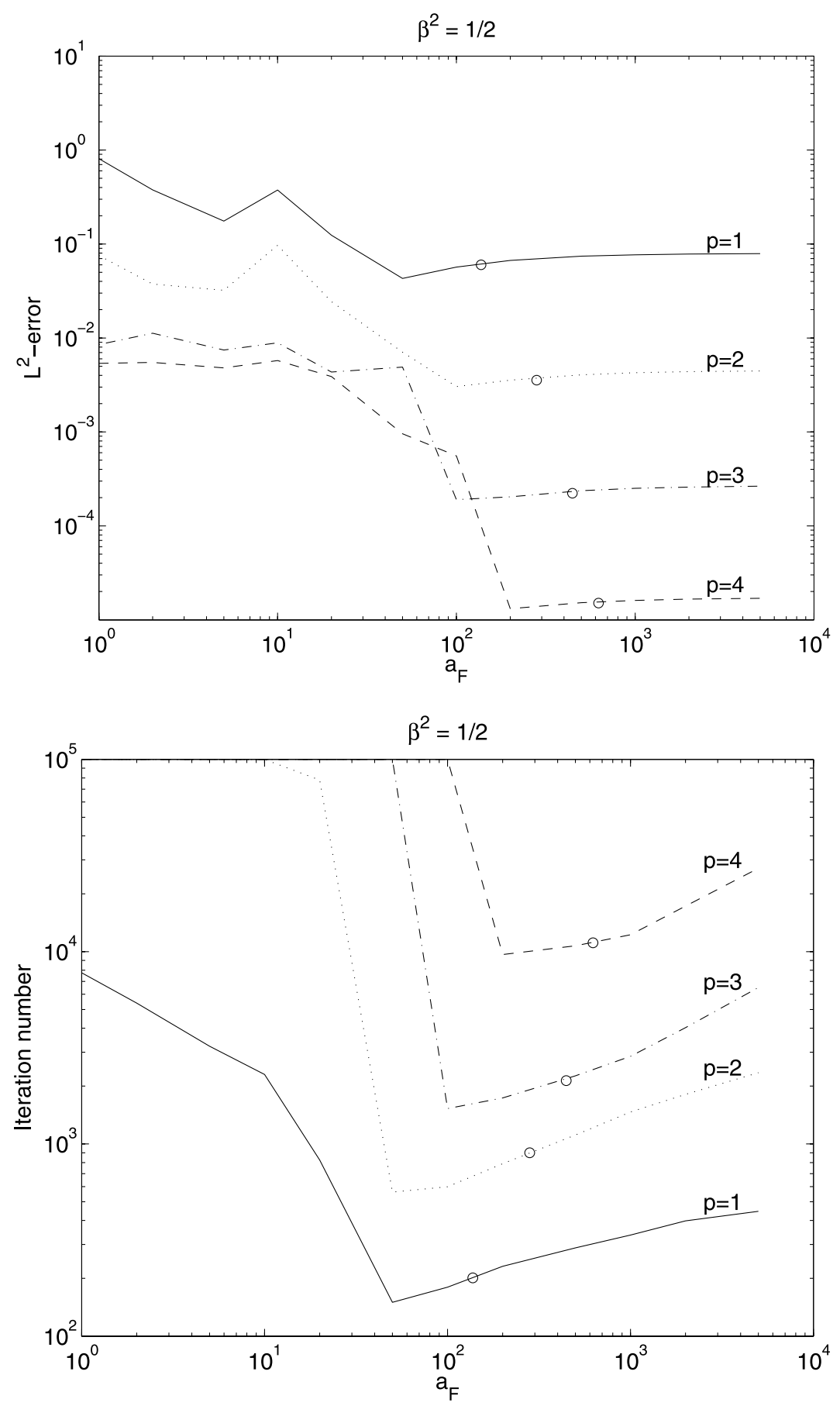

Fig. $3 L^{2}$-error (top) and the number of MINRES iterations (bottom) as a function of the penalty parameter $a_{F}$ in the IP-DG method. A structured mesh of 320 tetrahedra and coercivity constant $\beta^{2}=\frac{1}{2}$ are used. The circles indicate the theoretical estimate (71) 

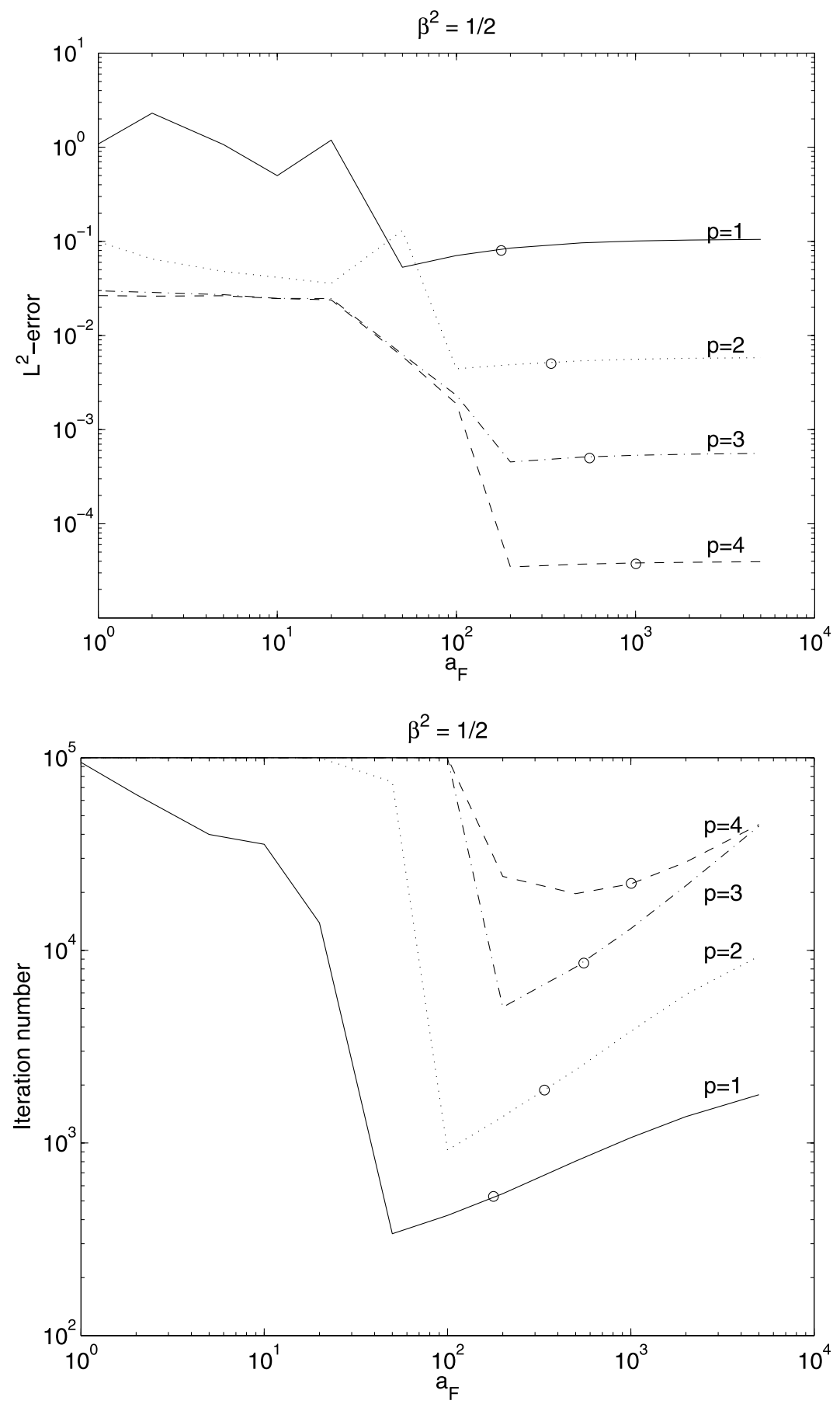

Fig. $4 L^{2}$-error (top) and the number of MINRES iterations (bottom) as a function of the penalty parameter $\mathrm{a}_{F}$ in the IP-DG method. An unstructured mesh of 432 tetrahedra and coercivity constant $\beta^{2}=\frac{1}{2}$ are used. The circles indicate the theoretical estimate (71) 
Table 1 Convergence of the IP-DG method on structured meshes

\begin{tabular}{|c|c|c|c|c|}
\hline & $\left\|\boldsymbol{E}-\boldsymbol{E}_{h}\right\|_{0}$ & Order & $\left\|\boldsymbol{E}-\boldsymbol{E}_{h}\right\|_{\mathrm{DG}}$ & Order \\
\hline \multicolumn{5}{|l|}{$p=1$} \\
\hline$N_{\mathrm{el}}=5$ & $2.4607 \mathrm{E}-01$ & - & $3.9831 \mathrm{E}-00$ & - \\
\hline$N_{\mathrm{el}}=40$ & 2.4279E-01 & 0.02 & $1.8609 \mathrm{E}-00$ & 1.10 \\
\hline$N_{\mathrm{el}}=320$ & $5.8254 \mathrm{E}-02$ & 2.06 & $9.8669 \mathrm{E}-01$ & 0.92 \\
\hline$N_{\mathrm{el}}=2560$ & $1.4962 \mathrm{E}-02$ & 1.96 & $5.0258 \mathrm{E}-01$ & 0.97 \\
\hline$N_{\mathrm{el}}=20480$ & $3.8285 \mathrm{E}-03$ & 1.97 & $2.5281 \mathrm{E}-01$ & 0.99 \\
\hline \multicolumn{5}{|l|}{$p=2$} \\
\hline$N_{\mathrm{el}}=5$ & 2.6774E-01 & - & $1.3192 \mathrm{E}-00$ & - \\
\hline$N_{\mathrm{el}}=40$ & $2.9019 \mathrm{E}-02$ & 3.21 & $4.9558 \mathrm{E}-01$ & 1.41 \\
\hline$N_{\mathrm{el}}=320$ & $3.5517 \mathrm{E}-03$ & 3.03 & $1.3257 \mathrm{E}-01$ & 1.90 \\
\hline$N_{\mathrm{el}}=2560$ & $4.5523 \mathrm{E}-04$ & 2.96 & 3.3900E-02 & 1.97 \\
\hline \multicolumn{5}{|l|}{$p=3$} \\
\hline$N_{\mathrm{el}}=5$ & $4.8262 \mathrm{E}-02$ & - & 8.8677E-01 & - \\
\hline$N_{\mathrm{el}}=40$ & $4.2128 \mathrm{E}-03$ & 3.52 & $9.9175 \mathrm{E}-02$ & 3.16 \\
\hline$N_{\mathrm{el}}=320$ & $2.2240 \mathrm{E}-04$ & 4.24 & $1.2976 \mathrm{e}-02$ & 2.93 \\
\hline$N_{\mathrm{el}}=2560$ & $1.3283 \mathrm{E}-05$ & 4.07 & $1.6408 \mathrm{E}-03$ & 2.98 \\
\hline \multicolumn{5}{|l|}{$p=4$} \\
\hline$N_{\mathrm{el}}=5$ & $2.2563 \mathrm{E}-02$ & - & $1.1561 \mathrm{E}-01$ & - \\
\hline$N_{\mathrm{el}}=40$ & $5.0697 \mathrm{E}-04$ & 5.48 & $1.5636 \mathrm{E}-02$ & 2.89 \\
\hline$N_{\mathrm{el}}=320$ & $1.5059 \mathrm{E}-05$ & 5.07 & $1.0211 \mathrm{E}-03$ & 3.94 \\
\hline
\end{tabular}

Table 2 Convergence of the IP-DG on unstructured meshes

\begin{tabular}{lllll}
\hline & $\left\|\boldsymbol{E}-\boldsymbol{E}_{h}\right\|_{0}$ & Order & $\left\|\boldsymbol{E}-\boldsymbol{E}_{h}\right\|_{\mathrm{DG}}$ & Order \\
\hline$p=1$ & & & & - \\
$N_{\mathrm{el}}=54$ & $2.1909 \mathrm{E}-01$ & - & $1.9906 \mathrm{E}-00$ & 0.87 \\
$N_{\mathrm{el}}=432$ & $7.6122 \mathrm{E}-02$ & 1.53 & $1.0922 \mathrm{E}-00$ & 0.90 \\
$N_{\mathrm{el}}=3456$ & $2.3563 \mathrm{E}-02$ & 1.69 & $5.8597 \mathrm{E}-01$ & 0.90 \\
$N_{\mathrm{el}}=27648$ & $7.3498 \mathrm{E}-03$ & 1.68 & $3.1488 \mathrm{E}-01$ & - \\
\hline$p=2$ & & & $4.2088 \mathrm{E}-01$ & 1.60 \\
$N_{\mathrm{el}}=54$ & $2.9574 \mathrm{E}-02$ & - & $1.3896 \mathrm{E}-01$ & 1.76 \\
$N_{\mathrm{el}}=432$ & $5.0225 \mathrm{E}-03$ & 2.56 & $4.0967 \mathrm{E}-02$ & - \\
$N_{\mathrm{el}}=3456$ & $7.4724 \mathrm{E}-04$ & 2.75 & & 2.51 \\
\hline$p=3$ & & & $1.1318 \mathrm{E}-01$ & 2.79 \\
$N_{\mathrm{el}}=54$ & $4.5752 \mathrm{E}-03$ & - & $1.9863 \mathrm{E}-02$ & - \\
$N_{\mathrm{el}}=432$ & $4.9868 \mathrm{E}-04$ & 3.20 & $2.8737 \mathrm{E}-03$ & 3.12 \\
$N_{\mathrm{el}}=3456$ & $3.8164 \mathrm{E}-05$ & 3.71 & & $1.2321 \mathrm{E}-02$ \\
\hline$p=4$ & & & $1.4133 \mathrm{E}-03$ & \\
$N_{\mathrm{el}}=54$ & $5.3253 \mathrm{E}-04$ & 3.83 & & \\
$N_{\mathrm{el}}=432$ & $3.7544 \mathrm{E}-05$ & & &
\end{tabular}


Table 3 Convergence of the method of DG method using the Brezzi formulation on structured meshes

\begin{tabular}{|c|c|c|c|c|}
\hline & $\left\|\boldsymbol{E}-\boldsymbol{E}_{h}\right\|_{0}$ & Order & $\left\|\boldsymbol{E}-\boldsymbol{E}_{h}\right\|_{\mathrm{DG}}$ & Order \\
\hline \multicolumn{5}{|l|}{$p=1$} \\
\hline$N_{\mathrm{el}}=5$ & $5.2748 \mathrm{E}-01$ & - & 4.2503E-00 & - \\
\hline$N_{\mathrm{el}}=40$ & 3.0757E-01 & 0.78 & $1.9832 \mathrm{E}-00$ & 1.10 \\
\hline$N_{\mathrm{el}}=320$ & $7.2366 \mathrm{E}-02$ & 2.09 & $1.0245 \mathrm{E}-00$ & 0.95 \\
\hline$N_{\mathrm{el}}=2560$ & 1.7809E-02 & 2.02 & $5.1664 \mathrm{E}-01$ & 0.99 \\
\hline$N_{\mathrm{el}}=20480$ & 4.4353E-03 & 2.01 & $2.5888 \mathrm{E}-01$ & 1.00 \\
\hline \multicolumn{5}{|l|}{$p=2$} \\
\hline$N_{\mathrm{el}}=5$ & 3.1495E-01 & - & $1.5266 \mathrm{E}-00$ & - \\
\hline$N_{\mathrm{el}}=40$ & $3.4580 \mathrm{E}-02$ & 3.19 & $5.2322 \mathrm{E}-01$ & 1.54 \\
\hline$N_{\mathrm{el}}=320$ & $4.2011 \mathrm{E}-03$ & 3.04 & $1.3581 \mathrm{E}-01$ & 1.95 \\
\hline$N_{\mathrm{el}}=2560$ & $5.2373 \mathrm{E}-04$ & 3.00 & $3.4936 \mathrm{E}-02$ & 1.96 \\
\hline \multicolumn{5}{|l|}{$p=3$} \\
\hline$N_{\mathrm{el}}=5$ & $6.4461 \mathrm{E}-02$ & - & 8.8839E-01 & - \\
\hline$N_{\mathrm{el}}=40$ & 4.7770E-03 & 3.75 & $1.0055 \mathrm{E}-01$ & 3.14 \\
\hline$N_{\mathrm{el}}=320$ & $2.4747 \mathrm{E}-04$ & 4.27 & $1.3053 \mathrm{E}-02$ & 2.95 \\
\hline$N_{\mathrm{el}}=2560$ & $1.4323 \mathrm{e}-05$ & 4.11 & $1.6458 \mathrm{e}-03$ & 2.99 \\
\hline \multicolumn{5}{|l|}{$p=4$} \\
\hline$N_{\mathrm{el}}=5$ & $2.3394 \mathrm{E}-02$ & - & $1.2148 \mathrm{E}-01$ & - \\
\hline$N_{\mathrm{el}}=40$ & $5.5416 \mathrm{E}-04$ & 5.40 & $1.5677 \mathrm{E}-02$ & 2.95 \\
\hline$N_{\mathrm{el}}=320$ & $1.6320 \mathrm{E}-05$ & 5.09 & $1.0238 \mathrm{E}-03$ & 3.94 \\
\hline
\end{tabular}

Table 4 Convergence of the DG method using the Brezzi formulation on unstructured meshes

\begin{tabular}{lllll}
\hline & $\left\|\boldsymbol{E}-\boldsymbol{E}_{h}\right\|_{0}$ & Order & $\left\|\boldsymbol{E}-\boldsymbol{E}_{h}\right\|_{\mathrm{DG}}$ & Order \\
\hline$p=1$ & & & & - \\
$N_{\mathrm{el}}=54$ & $3.0073 \mathrm{E}-01$ & - & $2.1553 \mathrm{E}-00$ & 0.88 \\
$N_{\mathrm{el}}=432$ & $9.4822 \mathrm{E}-02$ & 1.67 & $1.1568 \mathrm{E}-00$ & 0.91 \\
$N_{\mathrm{el}}=3456$ & $2.7757 \mathrm{E}-02$ & 1.77 & $6.1509 \mathrm{E}-01$ & 0.90 \\
$N_{\mathrm{el}}=27648$ & $8.3898 \mathrm{E}-03$ & 1.73 & $3.3034 \mathrm{E}-01$ & - \\
\hline$p=2$ & & & & 1.63 \\
$N_{\mathrm{el}}=54$ & $3.3899 \mathrm{E}-02$ & - & $1.4584 \mathrm{E}-01$ & 1.77 \\
$N_{\mathrm{el}}=432$ & $5.5733 \mathrm{E}-03$ & 2.60 & $4.2719 \mathrm{E}-02$ & - \\
$N_{\mathrm{el}}=3456$ & $8.1170 \mathrm{E}-04$ & 2.78 & & 2.52 \\
\hline$p=3$ & & & $1.1547 \mathrm{E}-01$ & 2.82 \\
$N_{\mathrm{el}}=54$ & $5.2999 \mathrm{E}-03$ & - & $2.0092 \mathrm{E}-02$ & 3.13 \\
$N_{\mathrm{el}}=432$ & $5.2979 \mathrm{E}-04$ & 3.32 & $2.8547 \mathrm{E}-03$ & \\
$N_{\mathrm{el}}=3456$ & $4.1110 \mathrm{E}-05$ & 3.69 & & \\
\hline$p=4$ & & & $1.2493 \mathrm{E}-02$ & $1.4241 \mathrm{e}-03$ \\
$N_{\mathrm{el}}=54$ & $5.6677 \mathrm{E}-04$ & - & & \\
$N_{\mathrm{el}}=432$ & $3.8693 \mathrm{e}-05$ & 3.87 & & \\
\hline & & & &
\end{tabular}


Based on the analysis in [18] and [10], the optimal convergence rate for this example is $\mathcal{O}\left(h^{p+1}\right)$ in the $L^{2}(\Omega)$-norm and $\mathcal{O}\left(h^{p}\right)$ in the DG norm. We can see that, for both methods on structured meshes, the optimal convergence rate is achieved in the $L^{2}(\Omega)$-norm, and higher-than-optimal convergence rates are observed in the DG norm. On unstructured meshes, we only have an estimated convergence rate with $h \sim N_{\mathrm{el}}^{-\frac{1}{3}}$. Here the convergence rates are slightly suboptimal, in part because we have to estimate the rates of convergence, and in part because we are still in the pre-asymptotic regime.

As a second example of asymptotic convergence, we solve the discrete eigenvalue problem that results from the DG approximation of (1) when the Brezzi type DG method (23) is used. All the eigenvalues of (1), corresponding to smooth eigenfunctions, are known to be

$$
\omega^{2}=\pi^{2}\left(l^{2}+m^{2}+n^{2}\right)
$$

where $l, m$ and $n$ are non-negative integers such that

$$
l m+l n+n m>0 .
$$

When $l m n>0$, there are two identical eigenvalues associated with linearly independent eigenfunctions. Again, the analysis in [9] provides a theoretical estimate for the convergence rate of the eigenvalues. That rate is $\mathcal{O}\left(h^{2 p}\right)$ for both methods described here, since the eigenspaces are smooth and the discretisations symmetric. Tables 5-8 show on a sequence of uniform meshes the first twenty exact and approximate eigenvalues, representing five different values because of the multiplicity. All eigenvalues are clearly free of spurious modes in this part of the spectrum. Actually, all eigenvalues whose eigenfunctions are reasonably well-resolved (e.g. relative $L^{2}$-error of 0.1 at most) are in the 'clear' spectrum

Table 5 Eigenvalues (divided by $\pi^{2}$ ) obtained on uniform meshes with $p=1$

\begin{tabular}{llllll}
\hline$h$ & $h / 2$ & $h / 4$ & $h / 8$ & $h / 16$ & Expected \\
\hline 3.1339 & 2.2578 & 2.0747 & 2.0192 & 2.0048 & 2.0000 \\
3.1339 & 2.2578 & 2.0747 & 2.0192 & 2.0048 & 2.0000 \\
3.1339 & 2.2578 & 2.0747 & 2.0192 & 2.0048 & 2.0000 \\
5.2780 & 3.7951 & 3.1682 & 3.0431 & 3.0108 & 3.0000 \\
5.7352 & 3.7951 & 3.1682 & 3.0431 & 3.0108 & 3.0000 \\
5.7352 & 5.5034 & 5.4426 & 5.1182 & 5.0300 & 5.0000 \\
8.5813 & 5.5034 & 5.4426 & 5.1182 & 5.0300 & 5.0000 \\
8.5813 & 5.5034 & 5.4426 & 5.1182 & 5.0300 & 5.0000 \\
8.5813 & 7.8215 & 5.4426 & 5.1182 & 5.0300 & 5.0000 \\
9.7578 & 7.8215 & 5.4426 & 5.1182 & 5.0300 & 5.0000 \\
9.7578 & 7.8215 & 5.4426 & 5.1182 & 5.0300 & 5.0000 \\
9.7578 & 8.2393 & 6.6343 & 6.1695 & 6.0430 & 6.0000 \\
11.7469 & 8.2393 & 6.6343 & 6.1695 & 6.0430 & 6.0000 \\
11.7469 & 8.2393 & 6.6343 & 6.1695 & 6.0430 & 6.0000 \\
11.7469 & 9.1638 & 6.6442 & 6.1707 & 6.0432 & 6.0000 \\
13.3385 & 9.1638 & 6.6442 & 6.1707 & 6.0432 & 6.0000 \\
17.2489 & 9.1638 & 6.6442 & 6.1707 & 6.0432 & 6.0000 \\
17.2489 & 12.2453 & 9.0311 & 8.2990 & 8.0768 & 8.0000 \\
17.2489 & 12.2453 & 9.0311 & 8.2990 & 8.0768 & 8.0000 \\
17.4002 & 12.2453 & 9.0311 & 8.2990 & 8.0768 & 8.0000 \\
\hline & & & & & \\
\hline
\end{tabular}


Table 6 Eigenvalues (divided by $\pi^{2}$ ) obtained on uniform meshes with $p=2$
Table 7 Eigenvalues (divided by $\pi^{2}$ ) obtained on uniform meshes with $p=3$

\begin{tabular}{|c|c|c|c|c|}
\hline$h$ & $h / 2$ & $h / 4$ & $h / 8$ & Expected \\
\hline 2.1270 & 2.0197 & 2.0014 & 2.0001 & 2.0000 \\
\hline 2.1270 & 2.0197 & 2.0014 & 2.0001 & 2.0000 \\
\hline 2.1270 & 2.0197 & 2.0014 & 2.0001 & 2.0000 \\
\hline 3.8664 & 3.0219 & 3.0047 & 3.0003 & 3.0000 \\
\hline 3.8664 & 3.0219 & 3.0047 & 3.0003 & 3.0000 \\
\hline 5.9288 & 5.1348 & 5.0196 & 5.0013 & 5.0000 \\
\hline 5.9288 & 5.1348 & 5.0196 & 5.0013 & 5.0000 \\
\hline 5.9288 & 5.1348 & 5.0196 & 5.0013 & 5.0000 \\
\hline 6.8030 & 5.2479 & 5.0196 & 5.0013 & 5.0000 \\
\hline 6.8030 & 5.2479 & 5.0196 & 5.0013 & 5.0000 \\
\hline 6.8030 & 5.2479 & 5.0196 & 5.0013 & 5.0000 \\
\hline 8.8557 & 6.3128 & 6.0335 & 6.0023 & 6.0000 \\
\hline 9.2855 & 6.3128 & 6.0335 & 6.0023 & 6.0000 \\
\hline 9.2855 & 6.3128 & 6.0335 & 6.0023 & 6.0000 \\
\hline 9.2855 & 6.4152 & 6.0352 & 6.0024 & 6.0000 \\
\hline 11.5504 & 6.4152 & 6.0352 & 6.0024 & 6.0000 \\
\hline 11.5504 & 6.4152 & 6.0352 & 6.0024 & 6.0000 \\
\hline 11.5504 & 8.5082 & 8.0789 & 8.0056 & 8.0000 \\
\hline 14.8586 & 8.5082 & 8.0789 & 8.0056 & 8.0000 \\
\hline 14.8586 & 8.5082 & 8.0789 & 8.0056 & 8.0000 \\
\hline
\end{tabular}

\begin{tabular}{|c|c|c|c|}
\hline$h$ & $h / 2$ & $h / 4$ & Expected \\
\hline 2.0482 & 2.0008 & 2.0000 & 2.0000 \\
\hline 2.0482 & 2.0008 & 2.0000 & 2.0000 \\
\hline 2.0482 & 2.0008 & 2.0000 & 2.0000 \\
\hline 3.1833 & 3.0067 & 3.0001 & 3.0000 \\
\hline 3.1833 & 3.0067 & 3.0001 & 3.0000 \\
\hline 5.2151 & 5.0236 & 5.0005 & 5.0000 \\
\hline 5.2151 & 5.0236 & 5.0005 & 5.0000 \\
\hline 5.2151 & 5.0236 & 5.0005 & 5.0000 \\
\hline 5.4621 & 5.0252 & 5.0005 & 5.0000 \\
\hline 5.4621 & 5.0252 & 5.0005 & 5.0000 \\
\hline 5.4621 & 5.0252 & 5.0005 & 5.0000 \\
\hline 6.6602 & 6.0446 & 6.0010 & 6.0000 \\
\hline 6.6602 & 6.0446 & 6.0010 & 6.0000 \\
\hline 6.6602 & 6.0446 & 6.0010 & 6.0000 \\
\hline 7.2220 & 6.0471 & 6.0011 & 6.0000 \\
\hline 7.2220 & 6.0471 & 6.0011 & 6.0000 \\
\hline 7.2220 & 6.0471 & 6.0011 & 6.0000 \\
\hline 8.9659 & 8.1927 & 8.0032 & 8.0000 \\
\hline 8.9659 & 8.1927 & 8.0032 & 8.0000 \\
\hline 10.4743 & 8.1927 & 8.0032 & 8.0000 \\
\hline
\end{tabular}


Table 8 Eigenvalues (divided by $\pi^{2}$ ) obtained on uniform meshes with $p=4$

\begin{tabular}{|c|c|c|c|}
\hline$h$ & $h / 2$ & $h / 4$ & Expected \\
\hline 2.0013 & 2.0000 & 2.0000 & 2.0000 \\
\hline 2.0013 & 2.0000 & 2.0000 & 2.0000 \\
\hline 2.0013 & 2.0000 & 2.0000 & 2.0000 \\
\hline 3.0118 & 3.0000 & 3.0000 & 3.0000 \\
\hline 3.0118 & 3.0000 & 3.0000 & 3.0000 \\
\hline 5.1823 & 5.0013 & 5.0000 & 5.0000 \\
\hline 5.1823 & 5.0013 & 5.0000 & 5.0000 \\
\hline 5.1823 & 5.0013 & 5.0000 & 5.0000 \\
\hline 5.1912 & 5.0014 & 5.0000 & 5.0000 \\
\hline 5.1912 & 5.0014 & 5.0000 & 5.0000 \\
\hline 5.1912 & 5.0014 & 5.0000 & 5.0000 \\
\hline 6.3525 & 6.0031 & 6.0000 & 6.0000 \\
\hline 6.3525 & 6.0031 & 6.0000 & 6.0000 \\
\hline 6.3525 & 6.0031 & 6.0000 & 6.0000 \\
\hline 6.4912 & 6.0037 & 6.0000 & 6.0000 \\
\hline 6.4912 & 6.0037 & 6.0000 & 6.0000 \\
\hline 6.4912 & 6.0037 & 6.0000 & 6.0000 \\
\hline 8.2632 & 8.0050 & 8.0001 & 8.0000 \\
\hline 8.8368 & 8.0050 & 8.0001 & 8.0000 \\
\hline 8.8368 & 8.0050 & 8.0001 & 8.0000 \\
\hline
\end{tabular}

for the parameter estimates derived in Sect. 4. The approximated eigenvalues converge asymptotically at a rate predicted by the theoretical results [9] and found in two-dimensional experiments [10].

\section{Concluding Remarks and Outlook}

We have derived optimal penalty parameters and error estimates for symmetric discontinuous Galerkin discretisations of the time-harmonic Maxwell equations. The penalty parameters are given so that the geometric information of the mesh and the polynomial order are taken into account and therefore they are valid in the pre-asymptotic regime. This contrasts with earlier results in the same field, which focused mainly on the asymptotic behaviour of the schemes. It is important that both the theoretical results and the ensuing numerical simulations consider finite mesh sizes in three dimensions, because in practice three-dimensional simulations are rarely asymptotic.

The numerical examples we have presented show that the theoretical estimates are sharper for higher-order polynomials in terms of computational work, and even in the worst case they are no more than 2-3 times more expensive than the best value that we found numerically. Finally, numerical convergence results are also provided to complement the existing theoretical and lower-dimensional numerical results in literature.

Acknowledgements This research was supported by the Dutch government through the national program BSIK: knowledge and research capacity, in the ICT project BRICKS (http://www.bsik-bricks.nl), theme MSV1. We are also grateful to Mike Botchev for his many useful comments. 
Open Access This article is distributed under the terms of the Creative Commons Attribution Noncommercial License which permits any noncommercial use, distribution, and reproduction in any medium, provided the original author(s) and source are credited.

\section{Appendix}

To obtain the fourth inequalities in Lemma 5, we rewrite the expression on the left-hand side of that inequality as

$$
(A \underline{\boldsymbol{u}}, \underline{\boldsymbol{v}}),
$$

where

$$
A=\left(\begin{array}{ccc}
k^{2} & 0 & 0 \\
0 & 1 & \mathcal{M} \\
0 & \mathcal{M} & \max _{F \in \mathcal{F}_{h}}\left(n_{f}+\eta_{F}\right) \mathcal{M}^{2}
\end{array}\right)
$$

and

$$
\begin{gathered}
\underline{\boldsymbol{u}}=\left(\|\boldsymbol{u}\|_{0},\left\|\nabla_{h} \times \boldsymbol{u}\right\|_{0},\left\|h_{F}^{-\frac{1}{2}} \llbracket \boldsymbol{u} \rrbracket_{T}\right\|_{0, \mathcal{F}_{h}}\right)^{T} \quad \Longrightarrow \quad|\underline{\boldsymbol{u}}|=\|\boldsymbol{u}\|_{\mathrm{DG}}, \\
\underline{\boldsymbol{v}}=\left(\|\boldsymbol{v}\|_{0},\left\|\nabla_{h} \times \boldsymbol{v}\right\|_{0},\left\|h_{F}^{-\frac{1}{2}} \llbracket \boldsymbol{v} \rrbracket_{T}\right\|_{0, \mathcal{F}_{h}}\right)^{T} \quad \Longrightarrow \quad|\underline{\boldsymbol{v}}|=\|\boldsymbol{v}\|_{\mathrm{DG}} .
\end{gathered}
$$

Since $A$ is symmetric we have

$$
(A \underline{\boldsymbol{u}}, \underline{\boldsymbol{v}}) \leq \max _{\lambda \in \operatorname{eig}(A)}|\lambda||\underline{\boldsymbol{u}}||\underline{\boldsymbol{v}}|=\max _{\lambda \in \operatorname{eig}(A)}|\lambda|\|\boldsymbol{u}\|\|\boldsymbol{v}\|,
$$

from which a straightforward computation gives

$$
\max _{\lambda \in \operatorname{eig}(A)}|\lambda|=\max \left\{k^{2}, \lambda_{2}\right\}
$$

where $\lambda_{2}$ is the solution of the equation

$$
0=\left(1-\lambda_{2}\right)\left(\max _{F \in \mathcal{F}_{h}}\left(n_{f}+\eta_{F}\right) \mathcal{M}^{2}-\lambda_{2}\right)-\mathcal{M}^{2} .
$$

Using (57) the larger solution can be estimated as

$$
\begin{aligned}
& \frac{1}{2} \max _{F \in \mathcal{F}_{h}}\left(n_{f}+\eta_{F}\right) \mathcal{M}^{2}+1 \\
& \quad+\frac{1}{2} \sqrt{\left(\max _{F \in \mathcal{F}_{h}}\left(n_{f}+\eta_{F}\right) \mathcal{M}^{2}+1\right)^{2}-4 \max _{F \in \mathcal{F}_{h}}\left(n_{f}+\eta_{F}\right) \mathcal{M}^{2}+4 \mathcal{M}^{2}} \\
& \leq \frac{1}{2}\left(\max _{F \in \mathcal{F}_{h}}\left(n_{f}+\eta_{F}\right) \mathcal{M}^{2}+1+\sqrt{\left(\max _{F \in \mathcal{F}_{h}}\left(n_{f}+\eta_{F}\right) \mathcal{M}^{2}+1\right)^{2}-4 \mathcal{M}^{2}+4 \mathcal{M}^{2}}\right) \\
& =\max _{F \in \mathcal{F}_{h}}\left(n_{f}+\eta_{F}\right) \mathcal{M}^{2}+1,
\end{aligned}
$$

hence

$$
\max _{\lambda \in \operatorname{eig}(A)}|\lambda| \leq \max _{F \in \mathcal{F}_{h}}\left\{k^{2},\left(n_{f}+\eta_{F}\right) \mathcal{M}^{2}+1\right\} .
$$


Similarly, the last inequality in Lemma 6 is obtained through defining the matrix

$$
B=\left(\begin{array}{ccc}
k^{2} & 0 & 0 \\
0 & 1 & 2 \mathcal{M} \\
0 & 2 \mathcal{M} & \max _{F \in \mathcal{F}_{h}} h_{F} \mathrm{a}_{F}
\end{array}\right),
$$

after which a simple calculation and using the inequality $h_{F} \mathrm{a}_{F} \geq 1$ yields

$$
\max _{\lambda \in \operatorname{eig}(B)}|\lambda|=\max _{F \in \mathcal{F}_{h}}\left\{k^{2}, h_{F} \mathrm{a}_{F}+\frac{3}{2} \mathcal{M}\right\} .
$$

\section{References}

1. Ainsworth, M., Coyle, J.: Hierarchic finite element bases on unstructured tetrahedral meshes. Int. J. Numer. Methods Eng. 58(14), 2103-2130 (2003)

2. Arnold, D.N., Brezzi, F., Cockburn, B., Marini, L.D.: Unified analysis of discontinuous Galerkin methods for elliptic problems. SIAM J. Numer. Anal. 39(5), 1749-1779 (2001)

3. Balay, S., Buschelman, K., Gropp, W.D., Kaushik, D., Knepley, M.G., McInnes, L.C., Smith, B.F., Zhang, H.: PETSc Web page (2001). http://www.mcs.anl.gov/petsc

4. Bassi, F., Rebay, S., Mariotti, G., Pedinotti, S., Savini, M.: A high order accurate discontinuous finite element method for inviscid and viscous turbomachinery flows. In: Proceedings of the 1997 2nd European Conference on Turbomachinery-Fluid Dynamics and Thermodynamics (Antwerpen, Belgium), pp. 99-108 (1997)

5. Bossavit, A.: A rationale for 'edge-elements' in 3-D fields computations. IEEE Trans. Magn. 24(1), 74-79 (1988)

6. Bossavit, A.: Solving Maxwell equations in a closed cavity, and the question of 'spurious modes'. IEEE Trans. Magn. 26(2), 702-705 (1990)

7. Bossavit, A.: On the representation of differential forms by potentials in dimension 3. In: Scientific computing in electrical engineering, Warnemünde, 2000. Lect. Notes Comput. Sci. Eng., vol. 18, pp. 97104. Springer, Berlin (2001)

8. Brezzi, F., Manzini, G., Marini, D., Pietra, P., Russo, A.: Discontinuous finite elements for diffusion problems. In: Atti Convegno in onore di F. Brioschi, Milan, 1997, pp. 197-217. Istitutto Lombardo, Accademia di Scienza e Lettere, Milan, Italy, 1999

9. Buffa, A., Perugia, I.: Discontinuous Galerkin approximation of the Maxwell eigenproblem. SIAM J. Numer. Anal. 44(5), 2198-2226 (2006)

10. Buffa, A., Houston, P., Perugia, I.: Discontinuous Galerkin computation of the Maxwell eigenvalues on simplicial meshes. J. Comput. Appl. Math. 204(2), 317-333 (2007)

11. Buffa, A., Perugia, I., Warburton, T.: The mortar-discontinuous Galerkin method for the 2D Maxwell eigenproblem. J. Sci. Comput. 40(1-3), 86-114 (2009). Available at http://www.springerlink. com/content/85055g4h55622j19/

12. Epshteyn, Y., Rivière, B.: Estimation of penalty parameters for symmetric interior penalty Galerkin methods. J. Comput. Appl. Math. 206(2), 843-872 (2007)

13. Ern, A., Guermond, J.-L.: Theory and Practise of Finite Elements. Applied Mathematical Sciences, vol. 159. Springer, New York (2004)

14. Hesthaven, J.S., Warburton, T.: Nodal high-order methods on unstructured grids. I. Time-domain solution of Maxwell's equations. J. Comput. Phys. 181(1), 186-221 (2002)

15. Hesthaven, J.S., Warburton, T.: High-order nodal discontinuous Galerkin methods for the Maxwell eigenvalue problem. Philos. Trans. R. Soc. Lond. Ser. A, Math. Phys. Eng. Sci. 362(1816), 493-524 (2004)

16. Hesthaven, J.S., Warburton, T.: Nodal Discontinuous Galerkin Methods. Texts in Applied Mathematics, vol. 54. Springer, New York (2008). Algorithms, analysis, and applications

17. Hiptmair, R.: Finite elements in computational electromagnetism. Acta Numer. 11, 237-339 (2002)

18. Houston, P., Perugia, I., Schneebeli, A., Schötzau, D.: Interior penalty method for the indefinite timeharmonic Maxwell equations. Numer. Math. 100(3), 485-518 (2005)

19. Houston, P., Perugia, I., Schötzau, D.: Mixed discontinuous Galerkin approximation of the Maxwell operator. SIAM J. Numer. Anal. 42(1), 434-459 (2004) 
20. Houston, P., Perugia, I., Schötzau, D.: Mixed discontinuous Galerkin approximation of the Maxwell operator: non-stabilized formulation. J. Sci. Comput. 22/23, 315-346 (2005)

21. Monk, P.: Finite Element Methods for Maxwell's Equations. Numerical Mathematics and Scientific Computation. Oxford University Press, New York (2003)

22. Nédélec, J.-C.: Mixed finite elements in $\mathbf{R}^{3}$. Numer. Math. 35(3), 315-341 (1980)

23. Nédélec, J.-C.: A new family of mixed finite elements in $\mathbf{R}^{3}$. Numer. Math. 50(1), 57-81 (1986)

24. Pesch, L., Bell, A., Sollie, H., Ambati, V.R., Bokhove, O., Van Der Vegt, J.J.W.: hpGEM-a software framework for discontinuous Galerkin finite element methods. ACM Trans. Math. Softw. 33(4), 23, 25 (2007)

25. Schötzau, D., Schwab, C., Toselli, A.: Stabilized $h p$-DGFEM for incompressible flow. Math. Models Methods Appl. Sci. 13(10), 1413-1436 (2003)

26. Shahbazi, K.: An explicit expression for the penalty parameter of the interior penalty method. J. Comput. Phys. 205(2), 401-407 (2005)

27. Šolín, P., Segeth, K., Doležel, I.: Higher-Order Finite Element Methods. Studies in Advanced Mathematics. Chapman \& Hall/CRC, Boca Raton (2004)

28. van der Vegt, J.J.W., Tomar, S.K.: Discontinuous Galerkin method for linear free-surface gravity waves. J. Sci. Comput. 22/23, 531-567 (2005)

29. Warburton, T., Embree, M.: The role of the penalty in the local discontinuous Galerkin method for Maxwell's eigenvalue problem. Comput. Methods Appl. Mech. Eng. 195(25-28), 3205-3223 (2006)

30. Warburton, T., Hesthaven, J.S.: On the constants in $h p$-finite element trace inverse inequalities. Comput. Methods Appl. Mech. Eng. 192(25), 2765-2773 (2003)

31. Whitney, H.: Geometric integration theory. Princeton University Press, Princeton (1957) 